University of Wollongong

Research Online

Faculty of Engineering and Information

Faculty of Engineering and Information

Sciences - Papers: Part B

Sciences

2019

Theoretical analysis of electrochemical noise measurement with single substrate electrode configuration and examination of the effect of reference electrodes

Thomas Jurak

University of Wollongong, Bluescope Limited, tj470@uowmail.edu.au

Sina S. Jamali

University of Wollongong, sjamali@uow.edu.au

Yue Zhao

University of Wollongong, yue@uow.edu.au

Follow this and additional works at: https://ro.uow.edu.au/eispapers1

Part of the Engineering Commons, and the Science and Technology Studies Commons

Research Online is the open access institutional repository for the University of Wollongong. For further information contact the UOW Library: research-pubs@uow.edu.au 


\title{
Theoretical analysis of electrochemical noise measurement with single substrate electrode configuration and examination of the effect of reference electrodes
}

\author{
Abstract \\ The development of the single substrate technique presents a realistic possibility of utilising \\ electrochemical noise measurement for on-site evaluations of corrosion behaviour. A theoretical model \\ was developed to further understand the effects of reference electrodes on the acquired data to increase \\ confidence in the technique. The model demonstrates that the reference electrodes can have a significant \\ impact on the measurements, resulting in erroneous values if the reference electrodes are not selected \\ carefully. Furthermore, the derived equations elucidate how to limit/remove such influence, facilitating \\ accurate application of the single substrate technique on bare metal and coated substrates both in \\ laboratories and in the field.

\section{Disciplines} \\ Engineering | Science and Technology Studies

\section{Publication Details} \\ Jurak, T., Jamali, S. S. \& Zhao, Y. (2019). Theoretical analysis of electrochemical noise measurement with \\ single substrate electrode configuration and examination of the effect of reference electrodes. \\ Electrochimica Acta, 301 377-389.
}




\title{
Theoretical Analysis of Electrochemical Noise Measurement with Single Substrate Electrode Configuration and Examination of the effect of Reference Electrodes
}

Thomas Jurak ${ }^{\mathrm{a}, \mathrm{b}, \mathrm{c}}$, Sina Jamali ${ }^{\mathrm{a}, \mathrm{b}}$, and Yue Zhao ${ }^{\mathrm{a}, \mathrm{b}}$

\footnotetext{
${ }^{a}$ Australian Research Council Research Hub for Australian Steel Manufacturing, University of Wollongong, Wollongong, Australia

${ }^{\mathrm{b}}$ School of Mechanical, Materials, Mechatronic and Biomedical Engineering, University of Wollongong, Wollongong, Australia

${ }^{\mathrm{c}}$ BlueScope Product Innovation and Technology, BlueScope Limited, Port Kembla, Australia
}

\begin{abstract}
The development of the single substrate technique presents a realistic possibility of utilising electrochemical noise measurement for on-site evaluations of corrosion behaviour. A theoretical model was developed to further understand the effects of reference electrodes on the acquired data to increase confidence in the technique. The model demonstrates that the reference electrodes can have a significant impact on the measurements, resulting in erroneous values if the reference electrodes are not selected carefully. Furthermore, the derived equations elucidate how to limit/remove such influence, facilitating accurate application of the single substrate technique on bare metal and coated substrates both in laboratories and in the field.
\end{abstract}

\section{Introduction}

Electrochemical noise measurement (ENM) of protective coatings has made significant progress since the pioneering works of Skerry and Eden in 1987-1991 [1,2]. Quick and simple acquisition and analysis of data combined with the advantage of not needing to polarise samples has led to significant diversification of how the technique is utilised [3-5]. 
Of great significance, a number of alternative electrode configurations have been established to date in an attempt to move ENM out of laboratories and into the field. These techniques are designed to facilitate the acquisition of in-situ quantitative electrochemical data of real world corrosion scenarios, particularly with a focus on industrial applications. This has been primarily investigated through two separate bodies of work. Firstly, there is the development and application of Electrochemical Emission Spectroscopy (EES), first proposed by Chen and Bogaerts [6], and advanced by Xia et.al [7-11]. EES utilises a platinum micro-cathode as the second working electrode for electrochemical noise measurements, in an attempt to eliminate the need for identical working electrodes that are impossible to achieve in reality. Secondly, there is the use of the single substrate (SS) and no-connection-to-substrate (NOCS) electrode configurations, advanced in the works of Mabbutt and Mills [5,12-15]. These alternative electrode configurations are the focus of the present work. Furthermore, there has been significant work directed towards theoretical validation of some of these techniques. In a review by Cottis on ENM techniques utilising asymmetrical working electrodes, it was concluded that under specific circumstances and with the correct assumptions, useful information about corrosion behaviour can be obtained [16]. The use of a platinum microcathode in EES was concluded to result in electrochemical current noise measurements being dictated by the high impedance of the micro-cathode, although electrochemical potential noise measurements were unaffected. In contrast, whilst the practical usage of the SS and NOCS electrode configurations has been demonstrated experimentally, to date there has not been a fundamental study of the theoretical aspects of either technique. Lastly, the Single Cell (SC) technique developed by Jamali et.al can be viewed as an extension to both prior works with asymmetrical working electrodes and the SS and NOCS techniques [17]. The SC technique requires only a single working electrode, with electrochemical potential and current noise data being recorded in sequence instead of simultaneously. Although a promising technique for in-situ ENM in its own right, the SC technique, as well as use of asymmetric working electrodes, will not be discussed in-depth here as our aim is to conduct a systematic analysis on the SS technique in this paper.

The SS technique differs from the well-established and standardised "salt bridge" (SB) configuration in three significant ways. Firstly, only a single connection to the substrate metal is required (hence the name), which is made to the reference electrode terminal of the measuring instrument. Secondly, two laboratory reference electrodes (LREs) are immersed in the corrosive electrolyte and are connected to the working electrode terminals of the 
measuring instrument. Thirdly, the electrical connection between the two cells is made via the metallic substrate and not via a salt bridge [5,12-15]. A comparison of the physical models of the SB and SS electrode configurations are outlined in Figures 1 and 2. It is worth noting that the NOCS technique takes the SS configuration a step further, replacing the connection to the substrate metal with a third LRE immersed in corrosive electrolyte in contact with the substrate metal. This third LRE is connected to the reference electrode terminal of the measuring instrument [5].

There is a complication in that connecting LREs to the working electrode terminals in the SS technique introduces two additional impedances to the equivalent electrical circuit (EEC) (as shown in Figure 3 for SB in comparison to Figure 4 for SS). In conventional direct current (DC) and alternating current (AC) electrochemical testing, it is recommended that the impedance of LREs be as low as possible [18-20]. Failure to maintain low reference electrode impedance can result in artefacts during AC electrochemical measurements, the effects of which are well documented [21-27]. The exact influence of connecting LREs to the working electrode terminals on acquired data through use of the SS technique has not been closely investigated from a theoretical perspective. It is sensible to assume that the impedance of any LREs used should be negligible relative to the impedance of the system being analysed, or their presence could have a significant impact on the ENM data. This has been considered previously by Mabbutt et.al [14]. It is a concern of the authors that even the use of well-maintained low impedance LREs will influence measurements of low impedance electrochemical systems via the SS technique, reducing confidence in the results. The SS technique has found favour amongst researchers performing electrochemical assessment of coated metallic substrates, but not bare metals. The effect of LREs on acquired data may be the root cause.

To date, researchers that have used the SS technique to assess corroding bare metals and coated metallic substrates have been able to empirically validate their results in the laboratory with other electrochemical techniques, lending credibility to the SS configuration [5,12-15,28-33]. It may be a safe assumption that the impedance of well-maintained LREs is negligible relative to a protective high impedance coating [14,15]. But a high impedance coating applied to a metallic substrate will inevitably degrade over time, as water and corrosive ions penetrate the coating, abrasion and weathering effects reduce its thickness, and under-film corrosion compromises adhesion. Thus it is also safe to assume that the service life of a given coating is a transition from being a protective, high impedance coating to a 
non-protective, low impedance coating. The time required to undergo this transition will vary widely, and will be dependent on the properties of the coating in question, the environment it is exposed to, and other factors. Therefore, the assumption of a high impedance coating may not be valid when the coating is analysed via ENM in the field, and verifying any in-field ENM data using alternative laboratory techniques is impractical if not impossible. Without knowing exactly what effect the LREs have on the acquired data, one cannot have confidence that valid data representative of the corroding system is being recorded. This is viewed by the authors as a significant hindrance to the widespread use of ENM in the field.

The authors present here a theoretical model for use of the SS technique of ENM based on a simple linear EEC. The analysis follows previous such efforts performed by Cottis et.al and Jamali et.al to elucidate the effects of solution resistance and coating impedance on ENM data respectively [34,35]. Experimental data demonstrating the effects of LRE impedance on a simple corrosion system of low carbon steel exposed to $3.5 \mathrm{wt} \% \mathrm{NaCl}$ solution is also presented. It is noteworthy that the focus of this manuscript is solely on examining the validity of data acquisition with the SS electrode configuration and not the statistical methods of data analysis.

\section{Theoretical Model}

As with the earlier efforts by Cottis et.al and Jamali et.al, it is important for the purposes of our analysis to break down the complex ENM setups into simple linear EECs, and clarify a number of underlying assumptions [34,35]. It is important to note that the EEC and subsequent analysis for the SB technique presented here is ultimately identical to the analysis presented in earlier publications for the purpose of determining the effect of coating impedance and solution resistance respectively on ENM data [34,35]. As such, the analysis presented here derives the same equations, and is included merely for the sake of convenience and ease of comparison to the analysis of the SS technique. A more detailed assessment of the effects of coating impedance and solution resistance on ENM data can be found in Ref. [34,35]. The physical systems analysed here are those presented in Figures 1 and 2; two identical coated metallic sections of equal size exposed to a specific quantity of corrosive electrolyte. In both setups, the sections of exposed metal are assumed to be identical in composition and surface condition, and the quantity and composition of corrosive 
electrolyte in each cell is assumed to be identical. Henceforth, all references of current noise and potential noise refer to the electrochemical current noise and electrochemical potential noise, respectively.

It is assumed that anodic and cathodic electrochemical reactions at the metal-solution interface are not hindered by the presence of the coating film, and hence the generation of electrochemical noise takes place independently of the coating. The solution resistance at the interface between the coating film and the metal is not considered in this model, as typically the solution is of very high ionic activity with very low resistance, and is assumed to be of constant composition for our analysis. Furthermore, whilst electrochemical reactions take place independently of the coating, the transport of corrosive ions through the coating film itself is assumed to be the rate-controlling process, and not the electrochemical reactions or the permeability of oxygen through the coating [35]. As demonstrated by Cottis et.al, both anodic and cathodic reactions at the metal-solution interface are assumed to be the singular source of current noise, whilst the potential noise is considered to be a response of the action of the current noise on the components of the electrical system in both setups [34]. Although the neutrality rule necessitates that the overall anodic and cathodic reactions are in balance and are therefore not independent, at any discrete time instance these reaction events are considered to be independent of each other and hence independent sources of current noise for the purposes of our analysis. The LREs used in both setups are assumed to be noiseless and identical, and therefore all potential noise is ultimately a consequence of electrochemical reactions at the exposed metal-solution interface. Furthermore, it is assumed that the source of the noise produced by the system is solely a consequence of the electrochemical reactions on the surface of the exposed metal, and not at all the result of mass transport events or the evolution of hydrogen gas such that bubbles are produced that alter the exposed surface area. A more detailed explanation of these assumptions can be found in previous works $[34,35]$. Finally, the exposed metal areas, electrolyte and LREs used in the SB setup are also assumed to be identical to those used in the SS setup.

In the SB technique of ENM, the measured current noise of one working electrode is the current signal from one electrode to the other. Assuming that the currents from two separate uncoated working electrodes are uncorrelated, negligible solution resistance, and identical working electrodes such that $\mathrm{Z}_{\mathrm{m} \text {-s.1 }}=\mathrm{Z}_{\mathrm{m} \text {-s.2 }}$, the current noise generated by working electrode 1 (WE1) will be halved, with one half flowing towards working electrode 2 (WE2) 
and the other half dissipating in electrochemical processes on WE1. Only the current that flows to WE2 is measured by the zero resistance ammeter (ZRA) as current noise power [34].

Therefore, based on these assumptions, the current noise power of each individual working electrode is:

$$
\overline{I_{n \text { measured }}^{2}}=\frac{\overline{I_{n}^{2}}}{4} \text { (eq.1) }
$$

Where: $I_{n \text { measured }}=$ current noise recorded by ZRA

$$
\overline{I_{n}^{2}}=\text { mean current noise or current noise power }
$$

Again, assuming currents are uncorrelated, the current noise powers add to give:

$$
\begin{gathered}
\overline{I_{n \text { measured }}^{2}}=\overline{\overline{I_{n}^{2}}} \frac{\overline{I_{n}^{2}}}{4} \\
\overline{I_{n \text { measured }}^{2}}=\overline{\frac{I_{n}^{2}}{2}} \text { (eq.2) }
\end{gathered}
$$

This has been demonstrated previously [34].

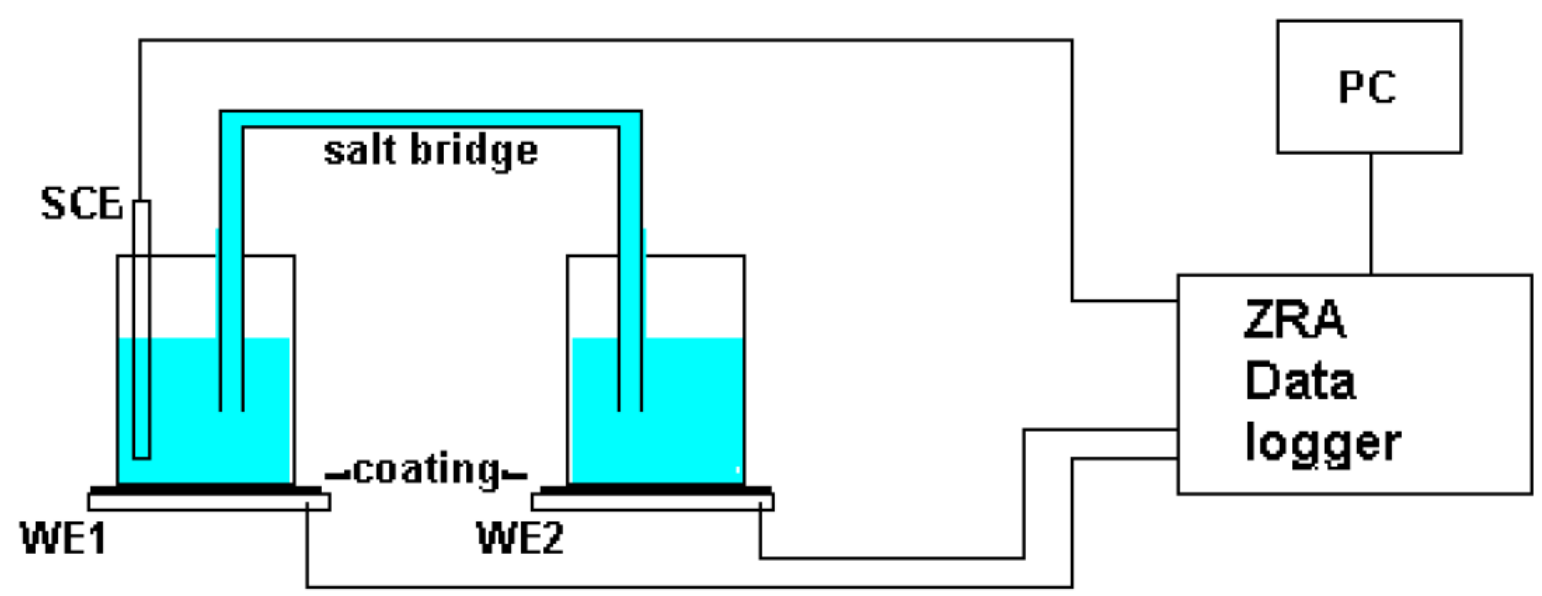

Figure 1: Diagram of the physical setup of the "salt bridge" technique of ENM. 


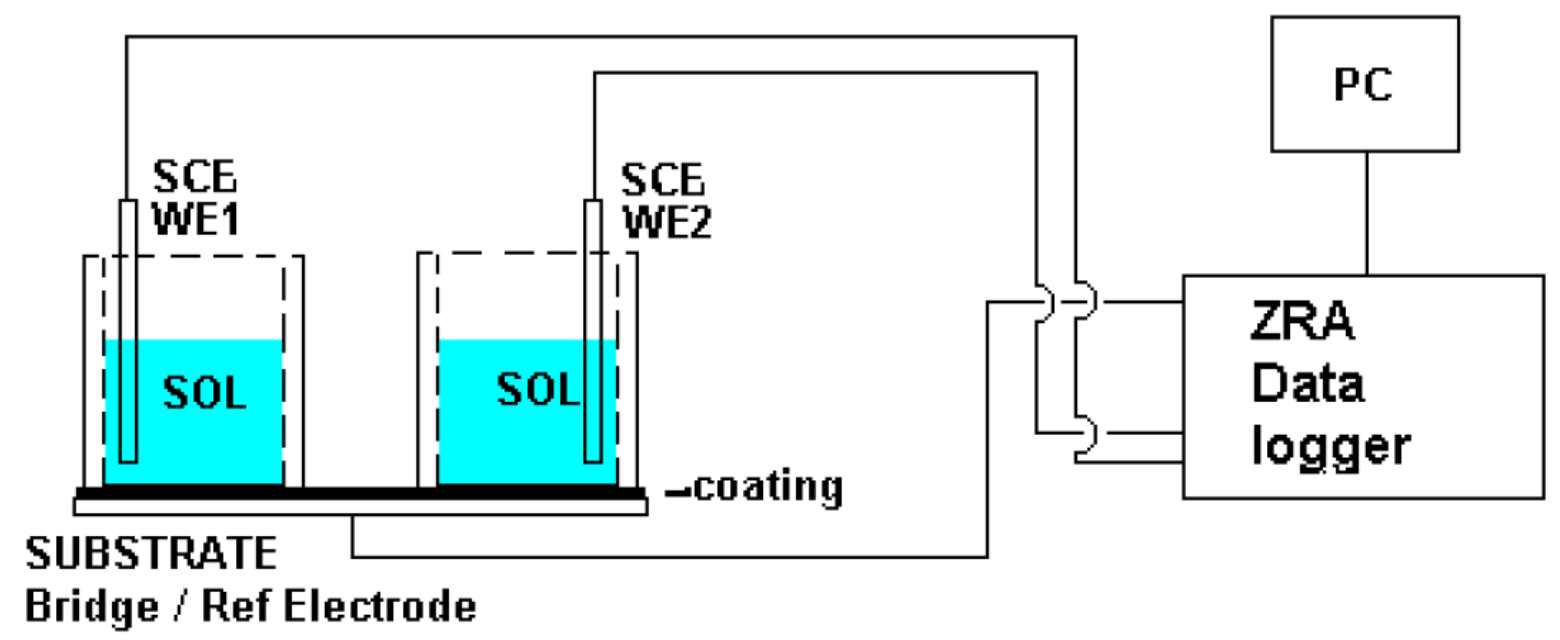

Figure 2: Diagram of the physical setup of the "single substrate" technique of ENM.

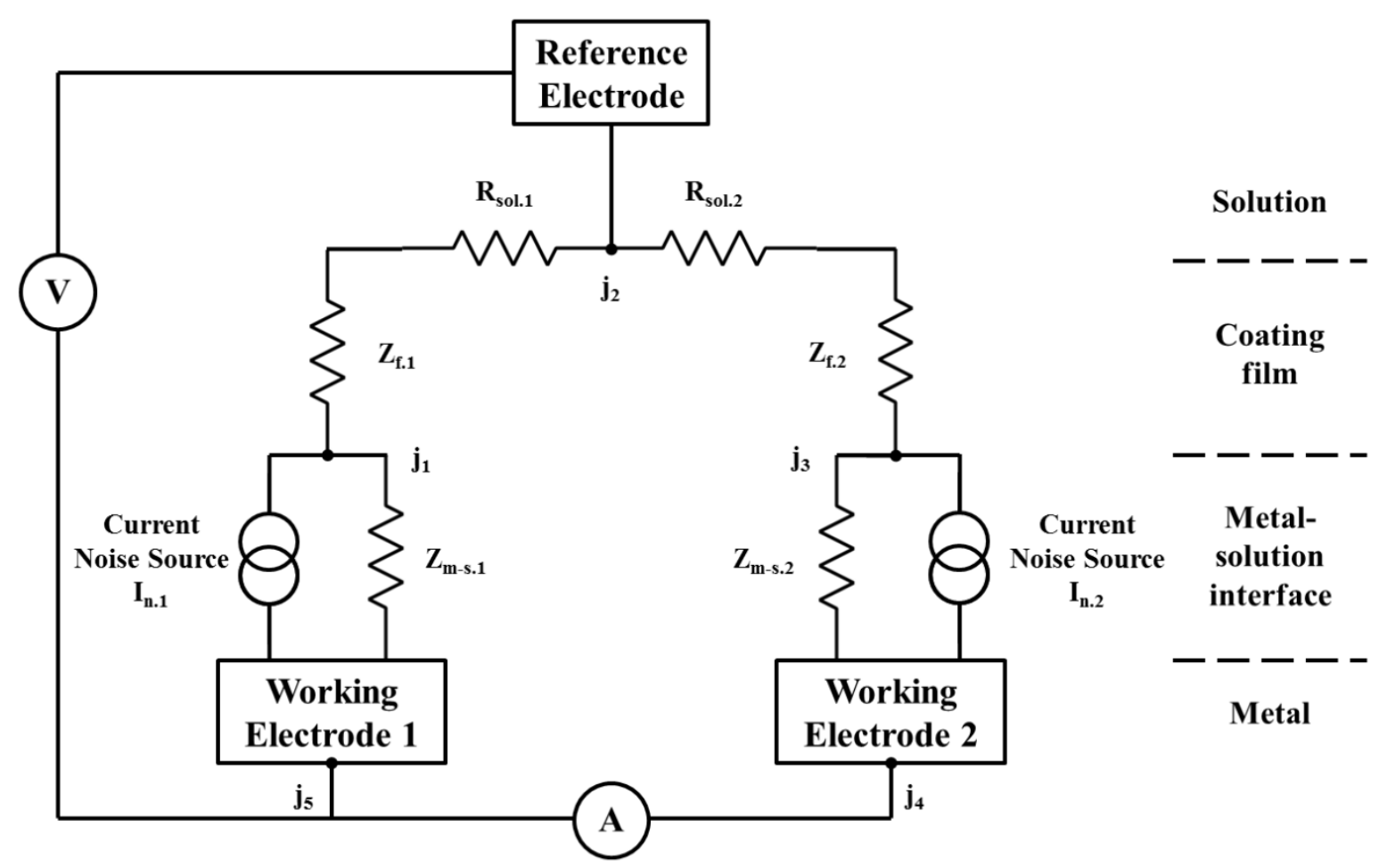

Figure 3: Equivalent electrical circuit diagram of the "salt bridge" technique of ENM. 


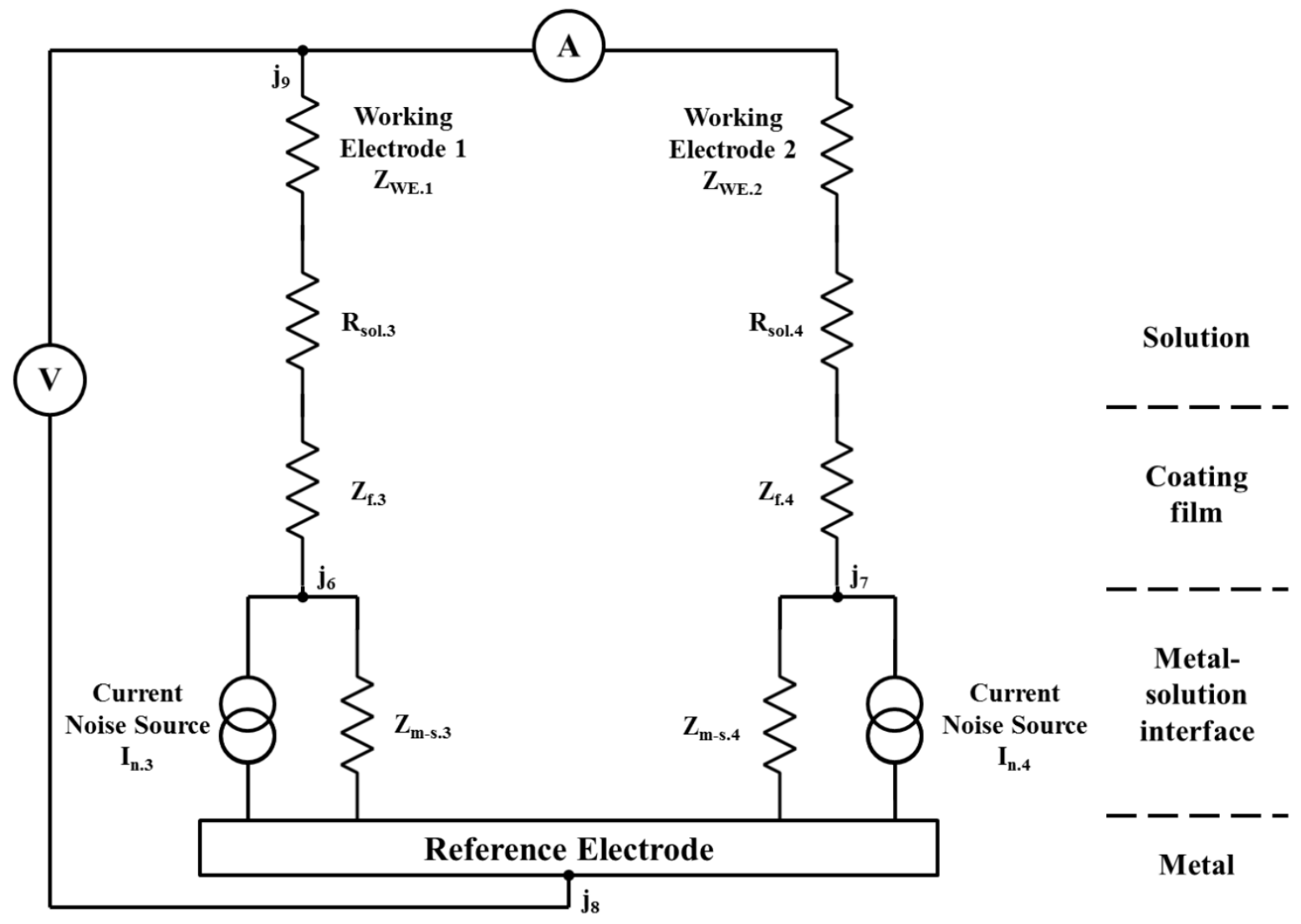

Figure 4: Equivalent electrical circuit diagram of the "single substrate" technique of ENM.

However, in the presence of significant solution resistance and a coating film, the current flowing from one electrode to the other will be attenuated. Therefore, the measured current noise power will be reduced. For example, considering the current noise source $\mathrm{I}_{\mathrm{n} .1}$ of WE1, the current will be split between the interfacial impedance $\mathrm{Z}_{\mathrm{m}-\mathrm{s} .1}$ and the series chain of $Z_{\mathrm{f} .1}+R_{\text {sol.1 }}+R_{\text {sol. } 2}+Z_{\mathrm{f} .2}+Z_{m-s .2}$ as outlined in Figure 5.

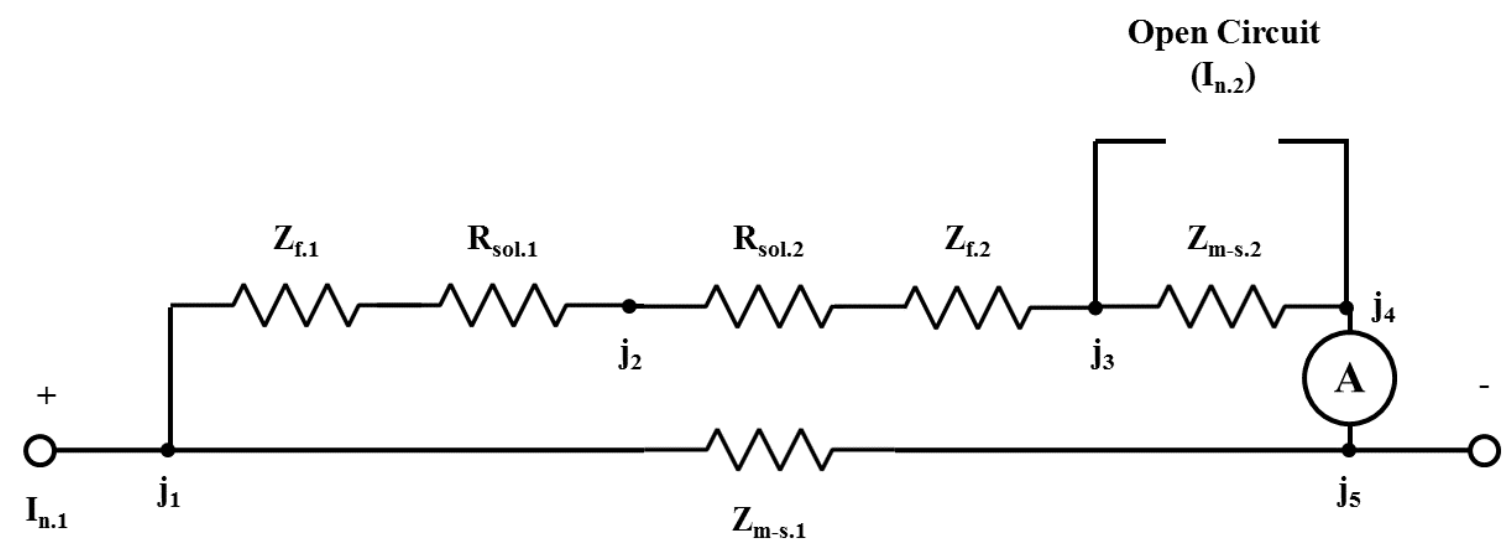

Figure 5: Equivalent electrical circuit diagram for current noise source $I_{n .1}$ for the "salt bridge" technique of ENM. 
Following our assumption that the current noise signals from two separate working electrodes are uncorrelated, the current noise powers add:

$$
\overline{I_{n \text { measured }}^{2}}=\left(\frac{\left(Z_{m-s .1} I_{n .1}\right)+\left(Z_{m-s .2} I_{n .2}\right)}{R_{\text {sol. } 1}+R_{\text {sol. } 2}+Z_{f .1}+Z_{f .2}+Z_{m-s .1}+Z_{m-s .2}}\right)^{2}
$$

Thus eq.3 gives the measured current noise power for the SB technique of ENM outlined in Figure 1 with dissimilar coated metallic sections, such that $\mathrm{Z}_{\mathrm{m} \text {-s.1 }} \neq \mathrm{Z}_{\mathrm{m} \text {-s.2 }}, \mathrm{I}_{\mathrm{n} .1} \neq$ $\mathrm{I}_{\mathrm{n} .2}$, and $\mathrm{Z}_{\mathrm{f} .1} \neq \mathrm{Z}_{\mathrm{f} .2}$, and with the LRE not equidistant between the two working electrodes $\left(\mathrm{R}_{\text {sol.1 }} \neq \mathrm{R}_{\text {sol. } 2}\right)$.

Assuming that the exposed metallic sections are identical such that $\mathrm{Z}_{\mathrm{m}-\mathrm{s} .1}=\mathrm{Z}_{\mathrm{m} \text {-s.2 }}=$ $\mathrm{Z}_{\mathrm{m}-\mathrm{s}}, \mathrm{I}_{\mathrm{n} .1}=\mathrm{I}_{\mathrm{n} .2}=\mathrm{I}_{\mathrm{n}}$, and $\mathrm{Z}_{\mathrm{f} .1}=\mathrm{Z}_{\mathrm{f} .2}=\mathrm{Z}_{\mathrm{f}}$, and assuming that the LRE is equidistant between the two working electrodes such that $\mathrm{R}_{\text {sol. } 1}=\mathrm{R}_{\text {sol. } 2}=\mathrm{R}_{\mathrm{sol}} / 2$ (or $\mathrm{R}_{\mathrm{sol} .1}+\mathrm{R}_{\text {sol. } 2}=\mathrm{R}_{\text {sol }}$ ), eq. 3 simplifies to:

$$
\overline{I_{n \text { measured }}^{2}}=2\left(\frac{Z_{m-s}}{R_{s o l}+2 Z_{f}+2 Z_{m-s}}\right)^{2} \overline{I_{n}^{2}}
$$

For uncoated metallic sections assessed using the $\mathrm{SB}$ technique, $\mathrm{Z}_{\mathrm{f}}=0$, and eq.4 simplifies to:

$$
\overline{I_{n \text { measured }}^{2}}=2\left(\frac{Z_{m-s}}{R_{\text {sol }}+2 Z_{m-s}}\right)^{2} \overline{I_{n}^{2}}(\mathbf{e q . 5})
$$

Finally, when solution resistance is negligible, such that $\mathrm{R}_{\text {sol }}=0$, eq.5 simplifies to eq.2. This is in agreement with earlier studies $[34,35]$.

For the SS technique, as for the SB technique, the current from one metallic section to another will be attenuated. Similarly, the measured current noise power will be reduced. For example, for the current noise source $I_{n .3}$, the current will be split between the interfacial impedance $Z_{\mathrm{m}-\mathrm{s} .3}$ and the series chain of $\mathrm{Z}_{\mathrm{f} .3}+\mathrm{R}_{\mathrm{sol} .3}+\mathrm{Z}_{\mathrm{WE} .1}+\mathrm{Z}_{\mathrm{WE} .2}+\mathrm{R}_{\mathrm{sol} .4}+\mathrm{Z}_{\mathrm{f} .4}+\mathrm{Z}_{\mathrm{m} \text {-s. } 4}$ as outlined in Figure 6. 


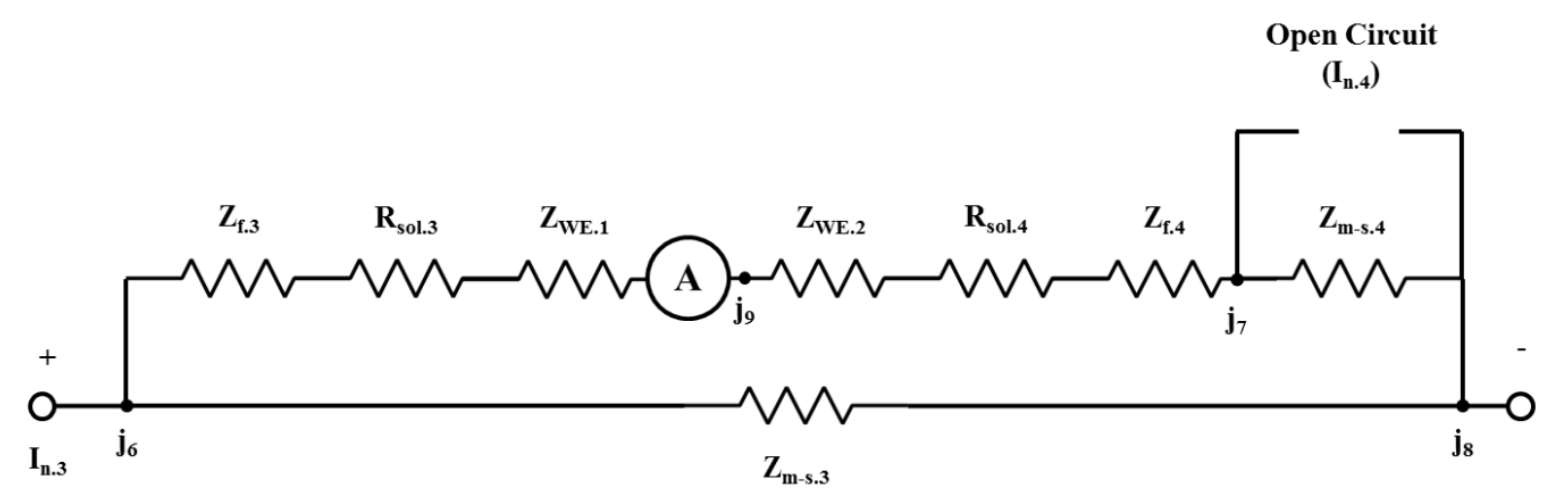

Figure 6: Equivalent electrical circuit diagram for current noise source $I_{n .3}$ for the "single substrate" technique of ENM.

Following our assumption that the current noise signals from two separate current noise sources are uncorrelated, the current noise powers add:

$$
\overline{I_{n \text { measured }}^{2}}=\left(\frac{\left(Z_{m-s .3} I_{n .3}\right)+\left(Z_{m-s .4} I_{n .4}\right)}{R_{\text {sol. } 3}+R_{\text {sol. } 4}+Z_{f .3}+Z_{f .4}+Z_{W E .1}+Z_{W E .2}+Z_{m-s .4}+Z_{m-s .3}}\right)^{2} \text { (eq.6) }
$$

Thus eq.6 gives the measured current noise power for the SS technique of ENM outlined in Figure 2 with dissimilar coated metallic sections, such that $\mathrm{Z}_{\mathrm{m} \text {-s.3 }} \neq \mathrm{Z}_{\mathrm{m} \text {-s. } 4}, \mathrm{I}_{\mathrm{n} .3} \neq$ $\mathrm{I}_{\mathrm{n} .4}$, and $\mathrm{Z}_{\mathrm{f} .3} \neq \mathrm{Z}_{\mathrm{f} .4}$, and dissimilar LREs $\left(\mathrm{Z}_{\mathrm{WE} .1} \neq \mathrm{Z}_{\mathrm{WE} .2}\right)$ not equidistant from their associated exposed metallic sections $\left(\mathrm{R}_{\text {sol. } 3} \neq \mathrm{R}_{\text {sol. } 4}\right)$.

Assuming that the exposed metallic sections are identical such that $\mathrm{Z}_{\mathrm{m}-\mathrm{s} .3}=\mathrm{Z}_{\mathrm{m} \text {-s. } 4}=$ $Z_{\mathrm{m}-\mathrm{s}}, \mathrm{I}_{\mathrm{n} .3}=\mathrm{I}_{\mathrm{n} .4}=\mathrm{I}_{\mathrm{n}}$, and $\mathrm{Z}_{\mathrm{f} .3}=\mathrm{Z}_{\mathrm{f} .4}=\mathrm{Z}_{\mathrm{f}}$, and assuming that the LREs connected to the working electrode terminals are both identical and equidistant from their associated exposed metallic sections such that $\mathrm{Z}_{\mathrm{WE} .1}=\mathrm{Z}_{\mathrm{WE} .2}=\mathrm{Z}_{\mathrm{WE}}$ and $\mathrm{R}_{\text {sol. } .3} / 2=\mathrm{R}_{\text {sol. } 4} / 2=\mathrm{R}_{\text {sol }}$, and eq. 6 simplifies to:

$$
\overline{I_{n \text { measured }}^{2}}=2\left(\frac{Z_{m-s}}{R_{s o l}+2 Z_{f}+2 Z_{W E}+2 Z_{m-s}}\right)^{2} \overline{I_{n}^{2}} \text { (eq.7) }
$$

For uncoated metallic sections assessed using the $\mathrm{SS}$ technique, $\mathrm{Z}_{\mathrm{f}}=0$, and eq.7 simplifies to:

$$
\overline{I_{n \text { measured }}^{2}}=2\left(\frac{Z_{m-s}}{R_{\text {sol }}+2 Z_{W E}+2 Z_{m-s}}\right)^{2} \overline{I_{n}^{2}} \text { (eq.8) }
$$

Of critical importance, assuming the use of ideal LREs with negligible (or near-zero) impedance, eq.7 simplifies to eq. 4 and eq. 8 simplifies to eq.5. As previously stated, when solution resistance is negligible, such that $\mathrm{R}_{\text {sol }}=0$, eq.5 simplifies to eq. 2 . 
Thus, assuming that the exposed metallic sections are identical, and assuming solution resistance is negligible as is often the case $\left(\mathrm{R}_{\mathrm{sol}}=0\right)$, it can be concluded that both the SB and SS techniques will measure the same current noise power only if the LREs connected to the working electrode terminals are ideal (and preferably identical), such that $\mathrm{Z}_{\mathrm{WE} .1}=\mathrm{Z}_{\mathrm{WE} .2}=0$, that is, their impedance is negligible. Mathematically:

$$
\overline{I_{n(S B)}^{2}}=\overline{I_{n(S S)}^{2}}
$$

Otherwise, the current noise signal is attenuated by the impedance of the LREs.

As clarified earlier, the current noise is produced by the electrochemical reactions as a consequence of the metallic section exposed to the corrosive electrolyte. The potential noise is produced by the current noise acting on the interfacial impedance $\left(\mathrm{Z}_{\mathrm{m}-\mathrm{s}}\right)$ of the exposed metallic section, in parallel with the solution resistance, coating film impedances and polarisation resistance of the second exposed section. In the absence of solution resistance and coating film impedance, the potential noise power for one current noise source is given by:

$$
\overline{E_{n}^{2}}=\frac{\overline{I_{n}^{2}} Z_{m-s}^{2}}{4}(\mathbf{e q . 9})
$$

Where: $\overline{E_{n}^{2}}=$ potential noise power

$$
\begin{aligned}
& \overline{I_{n}^{2}}=\text { mean current noise or current noise power } \\
& Z_{m-s}=\text { interfacial impedance at metal surface }
\end{aligned}
$$

This is in agreement with earlier studies [34,35].

To calculate potential noise power in the presence of solution resistance and coating film impedance for the SB technique, the effects of each source of current noise must be analysed independently, with all other voltage sources treated as short circuits, and all other current sources treated as open circuits. Consider the current noise source $\mathrm{I}_{n .1}$ of WE1. The current will be split between the interfacial impedance $Z_{m-s .1}$ and the series chain of $Z_{f .1}+$ $\mathrm{R}_{\mathrm{sol.1}}+\mathrm{R}_{\mathrm{sol.2}}+\mathrm{Z}_{\mathrm{f} .2}+\mathrm{Z}_{\mathrm{m}-\mathrm{s} .2}$ as outlined in Figure 5 . The potential will be measured at junction $\mathrm{j} .2$ between $\mathrm{R}_{\text {sol.1. }}$ and $\mathrm{R}_{\text {sol.2. }}$. Thus for $\mathrm{I}_{\mathrm{n} .1}$, using the potential dividing rule:

$$
E_{m-s .1}=\left(\frac{Z_{m-s .1}\left(R_{s o l .2}+Z_{f .2}+Z_{m-s .2}\right)}{R_{s o l .1}+R_{s o l .2}+Z_{f .1}+Z_{f .2}+Z_{m-s .2}+Z_{m-s .1}}\right) I_{n .1} \text { (eq.10) }
$$


Similarly, for $\mathrm{I}_{\mathrm{n} .2}$ :

$$
E_{m-s .2}=\left(\frac{Z_{m-s .2}\left(R_{s o l .1}+Z_{f .1}+Z_{m-s .1}\right)}{R_{s o l .1}+R_{s o l .2}+Z_{f .1}+Z_{f .2}+Z_{m-s .2}+Z_{m-s .1}}\right) I_{n .2} \text { (eq.11) }
$$

As per our earlier assumption, since the current noise sources are uncorrelated, the total potential noise power is given by:

$$
\overline{E_{n}^{2}}=\frac{\left(I_{n .1} Z_{m-s .1}\left(R_{s o l .2}+Z_{f .2}+Z_{m-s .2}\right)\right)^{2}+\left(I_{n .2} Z_{m-s .2}\left(R_{s o l .1}+Z_{f .1}+Z_{m-s .1}\right)\right)^{2}}{\left(R_{s o l .1}+R_{s o l .2}+Z_{f .1}+Z_{f .2}+Z_{m-s .2}+Z_{m-s .1}\right)^{2}} \text { (eq.12) }
$$

Thus eq.12 gives the measured potential noise power for the SB technique of ENM outlined in Figure 1 with dissimilar coated metallic sections, such that $\mathrm{Z}_{\mathrm{m} \text {-s.1 }} \neq \mathrm{Z}_{\mathrm{m} \text {-s.2 }}, \mathrm{I}_{\mathrm{n} .1} \neq$ $\mathrm{I}_{\mathrm{n} .2}$, and $\mathrm{Z}_{\mathrm{f} .1} \neq \mathrm{Z}_{\mathrm{f} .2 \text {. }}$

Assuming that the exposed metallic sections are identical, such that $\mathrm{Z}_{\mathrm{m}-\mathrm{s} .1}=\mathrm{Z}_{\mathrm{m}-\mathrm{s} .2}=$ $\mathrm{Z}_{\mathrm{m}-\mathrm{s}}, \mathrm{I}_{\mathrm{n} .1}=\mathrm{I}_{\mathrm{n} .2}=\mathrm{I}_{\mathrm{n}}$, and $\mathrm{Z}_{\mathrm{f} .1}=\mathrm{Z}_{\mathrm{f} .2}=\mathrm{Z}_{\mathrm{f}}$, and assuming the LRE is equidistant between the two working electrodes such that $\mathrm{R}_{\text {sol. } 1}=\mathrm{R}_{\text {sol. } 2}=\mathrm{R}_{\mathrm{sol}} / 2$ (or $\mathrm{R}_{\text {sol. } 1}+\mathrm{R}_{\text {sol. } 2}=\mathrm{R}_{\text {sol }}$ ), eq. 12 expands and simplifies to:

$$
\overline{E_{n}^{2}}=\frac{I_{n}^{2} z_{m-s}^{2}}{2}(\mathbf{e q . 1 3})
$$

Note that the potential noise power generated by two identical but separate current noise sources as expressed in eq.13 is double that generated by a single current noise source (eq.9). Thus for identical exposed metallic sections, electrochemical potential noise power is unaffected by solution resistance or coating film impedance. This has been demonstrated previously $[34,35]$.

Considering the SS technique, the potential noise is produced by the current noise acting on the interfacial impedance of the exposed metallic sections $\left(\mathrm{Z}_{\mathrm{m}-\mathrm{s}}\right)$ in parallel with the solution resistance, the coating film impedances, the impedances of the two LREs, and the polarisation resistance of the second exposed section. In the absence of these elements, the potential noise power for one current noise source is given by eq.9. As before, to calculate potential noise power in the presence of these elements, the effects of each source of current must again be analysed independently, with all other voltage sources treated as short circuits, and all other current noise sources treated as open circuits. Consider the current noise source $\mathrm{I}_{\mathrm{n} .3}$, the current will be split between the interfacial impedance $\mathrm{Z}_{\mathrm{m}-\mathrm{s} .3}$ and the series chain of $\mathrm{Z}_{\mathrm{f} .3}+\mathrm{R}_{\mathrm{sol.3}}+\mathrm{Z}_{\mathrm{WE} .1}+\mathrm{Z}_{\mathrm{WE} .2}+\mathrm{R}_{\mathrm{sol} .4}+\mathrm{Z}_{\mathrm{f} .4}+\mathrm{Z}_{\mathrm{m}-\mathrm{s} .4}$, as outlined in Figure 6. 
The potential will be measured at junction j.8 where $\mathrm{Z}_{\mathrm{m}-\mathrm{s} .4}$ meets $\mathrm{Z}_{\mathrm{m}-\mathrm{s} .3}$, as this junction is at the substrate metal which is what is physically connected to the reference electrode terminal of the measuring instrument. This is in turn electrically equivalent to measuring the potential at j.9, where the other end of the connection for potential measurement is made (i.e. potential is measured between junctions j.8 and j.9), between $\mathrm{Z}_{\mathrm{WE} .1}$

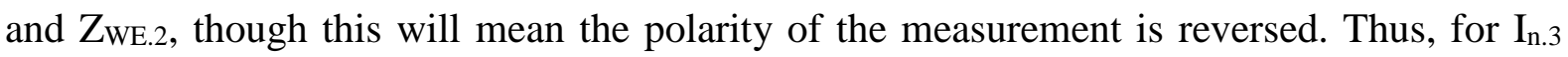
using the potential dividing rule:

$$
E_{m-s .3}=\left(\frac{-Z_{m-s .3}\left(Z_{W E .2}+R_{s o l .4}+Z_{f .4}+Z_{m-s .4}\right)}{R_{s o l .3}+R_{s o l .4}+Z_{f .3}+Z_{f .4}+Z_{W E .1}+Z_{W E .2}+Z_{m-s .3}+Z_{m-s .4}}\right) I_{n .3}
$$

Similarly, for $\mathrm{I}_{\mathrm{n} .4}$ :

$$
E_{m-s .4}=\left(\frac{-Z_{m-s .4}\left(Z_{W E .1}+R_{s o l .3}+Z_{f .3}+Z_{m-s .3}\right)}{R_{s o l .3}+Z_{W E .1}+Z_{f .3}+Z_{f .4}+Z_{W E .2}+R_{s o l .4}+Z_{m-s .3}+Z_{m-s .4}}\right) I_{n .4}
$$

As per our earlier assumption, since the current noise sources are uncorrelated, the total potential noise power is given by:

$$
\overline{E_{n}^{2}}=\frac{\left(-I_{n .3} Z_{m-s .3}\left(Z_{W E .2}+R_{s o l .4}+Z_{f .4}+Z_{m-s .4}\right)\right)^{2}+\left(-I_{n .4} Z_{m-s .4}\left(Z_{W E .1}+R_{s o l .3}+Z_{f .3}+Z_{m-s .3}\right)\right)^{2}}{\left(R_{s o l .3}+Z_{W E .1}+Z_{f .3}+Z_{f .4}+Z_{W E .2}+R_{s o l .4}+Z_{m-s .3}+Z_{m-s .4}\right)^{2}}
$$

(eq.16)

Eq.16 gives the measured potential noise power for the SS technique of ENM outlined in Figure 2 with dissimilar coated metallic sections, such that $Z_{\mathrm{m}-\mathrm{s} .3} \neq \mathrm{Z}_{\mathrm{m} \text {-s.4 }}, \mathrm{I}_{\mathrm{n} .3} \neq \mathrm{I}_{\mathrm{n} .4}$, and $\mathrm{Z}_{\mathrm{f} .3}$ $\neq \mathrm{Z}_{\mathrm{f} .4}$, and dissimilar LREs $\left(\mathrm{Z}_{\mathrm{WE} .1} \neq \mathrm{Z}_{\mathrm{WE} .2}\right)$ not equidistant from their associated exposed metallic sections $\left(\mathrm{R}_{\text {sol. } 3} \neq \mathrm{R}_{\text {sol. } 4}\right)$.

Assuming that the exposed metallic sections are identical, such that $\mathrm{Z}_{\mathrm{m}-\mathrm{s} .3}=\mathrm{Z}_{\mathrm{m}-\mathrm{s} .4}=$ $\mathrm{Z}_{\mathrm{m}-\mathrm{s}}, \mathrm{I}_{\mathrm{n} .3}=\mathrm{I}_{\mathrm{n} .4}=\mathrm{I}_{\mathrm{n}}$, and $\mathrm{Z}_{\mathrm{f} .3}=\mathrm{Z}_{\mathrm{f} .4}=\mathrm{Z}_{\mathrm{f}}$, and assuming that the LREs connected to the working electrode terminals are both identical and equidistant from their associated exposed metallic sections such that $\mathrm{Z}_{\mathrm{WE} .1}=\mathrm{Z}_{\mathrm{WE} .2}=\mathrm{Z}_{\mathrm{WE}}$ and $\mathrm{R}_{\mathrm{sol} .3} / 2=\mathrm{R}_{\text {sol. } 4} / 2=\mathrm{R}_{\mathrm{sol}}$, eq.16 expands and simplifies to:

$$
\overline{E_{n}^{2}}=\frac{I_{n}^{2} z_{m-s}^{2}}{2}(\mathbf{e q . 1 3})
$$

As stated previously, the potential noise power generated by two identical but separate current noise sources as expressed in eq.13 is double that generated by a single current noise source (eq.9). Thus, for identical exposed metallic sections with coating films, 
electrochemical potential noise power is unaffected by solution resistance, coating film impedance or any impedance introduced by connecting LREs to the working electrode terminals. Mathematically:

$$
\overline{E_{n(S B)}^{2}}=\overline{E_{n(S S)}^{2}}
$$

As explained earlier and outlined in eq.14 and eq.15, the polarity of any measured potential noise will be reversed, as may be expected intuitively since the current noise sources are connected to the reference electrode terminal of the measuring instrument. This has been demonstrated experimentally in the literature [5,12-15] and is again demonstrated experimentally in Section 4.3. Mathematically:

$$
\begin{aligned}
& E_{n(S S)}=E_{m-s .3}+E_{m-s .4} \\
& E_{n(S B)}=E_{m-s .1}+E_{m-s .2}
\end{aligned}
$$

According to eq.14 and eq.15, $\mathrm{E}_{\mathrm{m}-\mathrm{s} .3}$ and $\mathrm{E}_{\mathrm{m} \text {-s. } .4}$ are negative values due to the polarity reversal, and since potential noise power is unaffected by solution resistance, coating film impedance or the impedance of the LREs, we can conclude:

$$
E_{n(S B)}=-E_{n(S S)}
$$

Noise resistance is defined by the ratio of the standard deviations of the potential and current noise [21]. Mathematically:

$$
R_{n}=\frac{\sigma_{v}}{\sigma_{i}}(\mathbf{e q . 1 7})
$$

For the SB technique, substituting $\sigma_{\mathrm{v}}$ with eq.13 and $\sigma_{\mathrm{i}}$ with eq.4:

$$
R_{n}=\frac{R_{s o l}}{2}+Z_{f}+Z_{m-s} \text { (eq.18) }
$$

For uncoated metallic sections assessed using the $\mathrm{SB}$ technique, $\mathrm{Z}_{\mathrm{f}}=0$, and eq.18 simplifies to:

$$
R_{n}=\frac{R_{s o l}}{2}+Z_{m-s} \text { (eq.19) }
$$

Thus in the presence of negligible solution resistance, $R_{n}=Z_{m-s}$, or rather noise resistance is equal to the interfacial impedance of the metallic sections being analysed. This has been demonstrated previously [35]. 
For the SS technique, substituting $\sigma_{\mathrm{v}}$ with eq. 13 and $\sigma_{\mathrm{i}}$ with eq.7, we get:

$$
R_{n}=\frac{R_{s o l}}{2}+Z_{f}+Z_{W E}+Z_{m-s}(\text { eq.20) }
$$

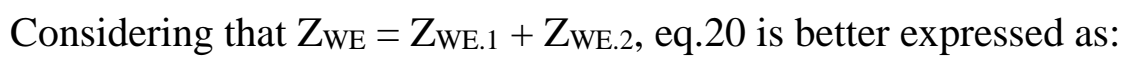

$$
R_{n}=\frac{R_{S o l}}{2}+\frac{Z_{W E .1}+Z_{W E .2}}{2}+Z_{f}+Z_{m-s} \text { (eq.21) }
$$

For uncoated metallic samples assessed using the $\mathrm{SS}$ technique, $\mathrm{Z}_{\mathrm{f}}=0$, and eq. 21 simplifies to:

$$
R_{n}=\frac{R_{S o l}}{2}+\frac{Z_{W E .1}+Z_{W E .2}}{2}+Z_{m-s} \text { (eq.22) }
$$

In the presence of negligible solution resistance, and assuming ideal (and identical) LREs connected to the working electrode terminals, eq. 22 simplifies to $R_{n}=Z_{m-s}$, noise resistance being equal to the interfacial impedance of the metallic sections being analysed. Thus, based on these assumptions:

$$
R_{n(S B)}=R_{n(S S)}
$$

Therefore, it can be concluded that when using ideal LREs, the SB and SS techniques measure the same electrochemical phenomena and will produce identical results, with the exception that the potential polarity will be reversed. Otherwise, the impedance of the LREs adds to the overall noise resistance.

There has been well documented usage of the SS and NOCS techniques in the literature to date [5,12-15,28-33]. However, all of these investigations conclude that the choice of LRE, or pseudo-reference electrode in the case of platinum foil, has no significant effect on the acquired ENM data. It is clear from eq. 21 and eq. 22 that this is only true for LREs with negligible impedance compared to that of the electrochemical system being examined. In Section 4, the effects of significant LRE impedance when utilising the SS technique is demonstrated experimentally. 


\section{Consequences of the Theoretical Model}

It is recommended that the impedance of LREs be as low as possible for both DC and AC electrochemical tests [18-20]. Ideally, the impedance of LREs should in fact be zero in order to make the best use of the sensitivity of the recording instrument, though in reality this is not possible $[18,19]$. However, since many electrochemical tests necessitate the use of a high impedance input voltmeter to measure electrode potentials via a LRE, the impact of reference electrode impedance is not widely understood, and is frequently ignored $[19,20]$. Utilising LREs for ENM via the SS technique without understanding and measuring the impedance of the recording electrodes could lead to significant error, as outlined by eq.21 and eq. 22 .

When utilising the SS technique, the impedance of the LREs used should be negligible relative to the impedance of the system being analysed. This is easier to achieve when assessing protective organic coatings over metallic substrates as outlined by Mabbutt et.al, since the impedance of the system being analysed is almost certain to be much higher relative to the impedance of a $\operatorname{LRE}$ (e.g. $1-100 \mathrm{M} \Omega$ compared to $1 \mathrm{k} \Omega$ for a "good" LRE) [14]. However, there are instances where the SS technique has been utilised to assess low impedance/resistance systems $(\sim 10 \mathrm{k} \Omega)$, such as uncoated metals or damaged/scribed coatings over metallic substrates, with no consideration for what influence the LREs may have upon noise resistance measurements $[5,12,13,15]$. The authors do not suggest that such measurements may be incorrect. In all cases any data obtained with the SS technique has been verified using other electrochemical analysis techniques. It is erroneous however to simply assume that the impedance contribution of a LRE is negligible in relation to the impedance of the system being analysed. Each LRE will contribute to the impedance of the circuit when utilizing the SS technique, as dictated by eq.21 and eq.22. Furthermore, using LREs to record electrochemical current noise data via the SS technique for low impedance/resistance systems may pose additional problems. Specifically, a higher flow of current through the LREs may affect their stability, and the exact effects of this on recorded data it is not known at present.

In order to circumvent this limitation of the SS technique and increase confidence in ENM data, as a minimum, the impedance of any LRE used should be measured in the laboratory prior to performing ENM in the field. When presenting noise resistance data, use 
of the SS technique should be made clear, and the impedance of the LREs used should also be reported and compared with the noise resistance $\left(R_{n}\right)$ values obtained.

Alternatively, inert metallic electrodes such as platinum foil can be used as pseudoreference electrodes instead of LREs to acquire ENM data using the SS technique. Several researchers have demonstrated this as a viable method in the literature through the use of platinum foil electrodes embedded in organic coatings over metallic substrates [28-33]. In each instance, the researchers positioned a platinum foil electrode between the organic coating primer and topcoat, electrically isolating the foil from the metallic substrate and the external environment. These platinum foil sections were then connected to the working electrode terminals of the measuring instrument, whilst a single electrical connection from the metallic substrate was made to the reference electrode terminal. All researchers reported good correlation between ENM data obtained in this way and ENM data obtained by conventional means [28-33]. It is important to note that whilst the authors expect a minimal impedance contribution from platinum electrodes on ENM data, and whilst the experimental data referenced here appears to support this, the effects of pseudo-reference electrode impedance on ENM data for platinum foil electrodes has not been discussed or investigated specifically to date.

Mabbutt et.al investigated the influence of different LREs and pseudo-reference electrodes specifically for the SS and NOCS electrode configurations, although the motivation for doing so was due to concerns over the noise intrinsic to LREs contributing to the measured noise data, and not due to the influence of LRE impedance [5]. Mabbutt conceived that when utilising the SS and NOCS techniques there are more sources of noise owing to the use of two or three LREs for the techniques respectively. Whilst ideally LREs should be noiseless, and are frequently assumed to be so, in reality this is not true $[4,21,34]$. Saturated calomel electrodes (SCEs), silver/silver chloride $(\mathrm{Ag} / \mathrm{AgCl})$ electrodes and platinum foil electrodes were investigated. It was concluded that choice of recording electrode was independent of the $R_{n}$ for each coated sample measured via the NOCS technique [5]. Though not discussed, this result implies that the LRE's used had negligible impedance relative to the coating systems analysed.

There is one final consequence of the theoretical model. Concerning the SB technique, it is clear from previous work that it is the current noise source and metal-solution interface impedance produced by the two exposed metallic sections that must be identical, or 
approximately so, in order for the calculated noise resistance $\left(\mathrm{R}_{\mathrm{n}}\right)$ to be inversely proportional to corrosion rate [36,37]. These metallic sections are identified as the working electrodes, and as stated previously, identical working electrodes are not possible in reality. There have been claims that data acquired using the SS technique is of noticeably improved accuracy, owing to the use of more identical working electrodes in the form of LREs $[12,14,15]$. The authors dispute this claim, as there is no theoretical or mathematical basis to support it. Considering the SS technique, it is again the current noise source and metal-solution interface impedance produced by the two exposed metallic sections that must be nominally identical. The fact that this coupled pair are identified as the reference electrode whilst LREs are identified as the working electrodes does not change the theoretical requirements of identical current noise sources. In addition, the LREs used should be as close to identical and "ideal" as possible, i.e., they should both have a stable and identical potential, contribute negligible noise to the electrochemical system, and have negligible or near-zero impedance, as stated earlier. There have also been claims that data acquired using the SS method contains less DC drift than data acquired using the SB method [12]. This is likely due to the two current noise sources being electrically connected (being the same section of material) prior to ENM, whereas two separate samples are required for the SB method. This could be remedied by electrically connecting the metallic section working electrodes of the SB method for a time prior to ENM.

\section{Experimental Analysis of Reference Electrodes in Single Substrate Configuration}

\subsection{Outline}

ENM was performed utilising the SS technique on low carbon steel S-type Q-panels in $3.5 \mathrm{wt} \% \mathrm{NaCl}$ solution. Various LREs were tested in order to demonstrate the effects of electrode impedance on ENM data and verify the theoretical model and its consequences. 


\subsection{Experiment Details}

The surface tested was a standard low carbon steel S-type Q-panel. The corrosive electrolyte used was a $3.5 \mathrm{wt} \% \mathrm{NaCl}$ solution. An uncoated, S-type Q-panel exposed to $3.5 \mathrm{wt} \% \mathrm{NaCl}$ solution was chosen in order to produce a worst case scenario for use of the SS technique. Corrosion of unprotected and uncoated carbon steel in $3.5 \mathrm{wt} \% \mathrm{NaCl}$ solution would be expected to produce a relatively high corrosion current, and hence a high current noise signal. This in turn constitutes a low impedance system, making it easier to identify the effects of LREs with high impedance, as well as providing greater delineation between different LREs. The composition of the alloy was as follows: $0.15 \mathrm{wt} \% \mathrm{C}, 0.6 \mathrm{wt} \% \mathrm{Mn}$, $0.03 \mathrm{wt} \% \mathrm{P}$ and $0.035 \mathrm{wt} \% \mathrm{~S}$. All samples were stored wrapped in paper containing a vapour phase rust inhibitor. All samples were rinsed with acetone, deionised (DI) water and then ethanol prior to testing.

ENM was performed utilising the SS technique with a number of different LREs. The LREs tested included; mercury/mercurous sulphate $\left(\mathrm{Hg}_{2} \mathrm{SO}_{4}\right)$ electrodes supplied by $\mathrm{CH}$ Instruments, Inc. (part number CHI151), and SCEs supplied by Sentek (R1 reference electrode). The complete list of LREs used is summarised in Table 1. Examples of these LREs can be seen in Figure 7.

Table 1: Details of laboratory reference electrodes used.

\begin{tabular}{|c|c|c|}
\hline Electrode ID & Electrode Type & Supplier \\
\hline SCE1 & mercury/mercury chloride & Sentek \\
\hline SCE2 & mercury/mercury chloride & Sentek \\
\hline Ref.E3 & mercury/mercurous sulphate & CHI, Inc. \\
\hline Ref.E4 & mercury/mercurous sulphate & CHI, Inc. \\
\hline Ref.E5 & mercury/mercurous sulphate & CHI, Inc. \\
\hline Ref.E6 & mercury/mercurous sulphate & CHI, Inc. \\
\hline
\end{tabular}




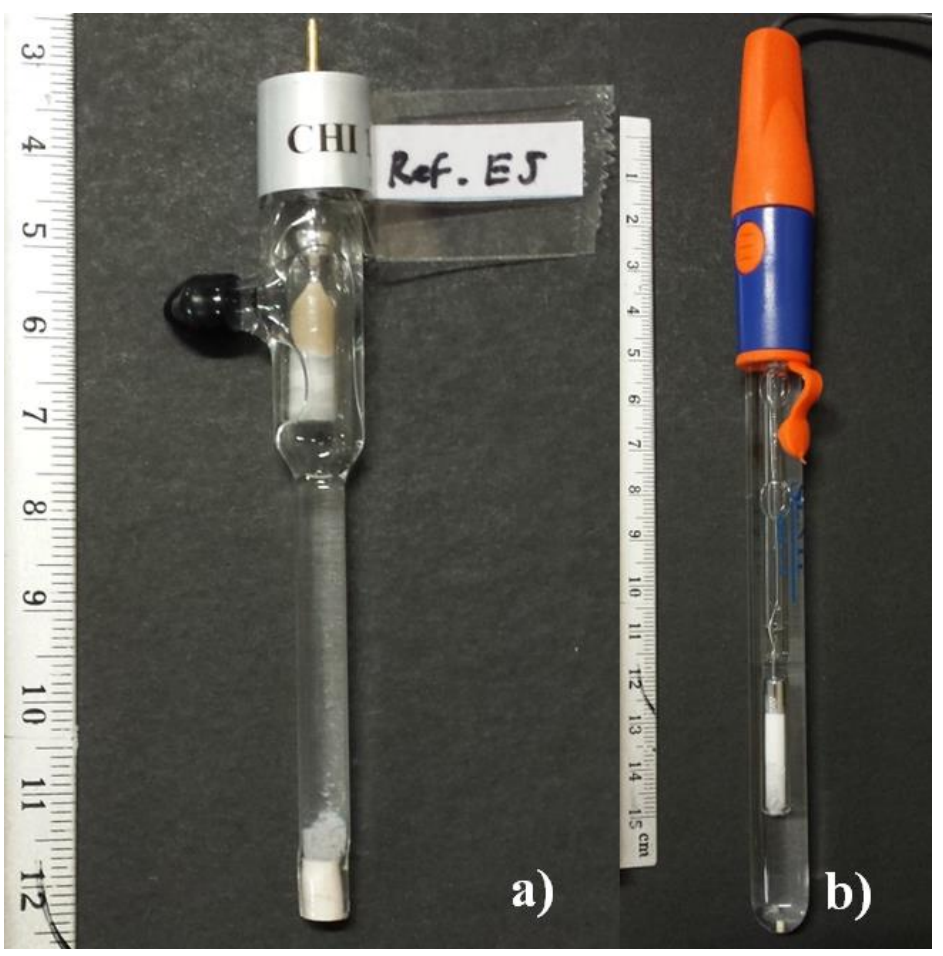

Figure 7: Photograph showing a) mercury/mercurous sulphate electrode and b) SCE.

An in-house built Perspex cell setup was held to the Q-panel samples using spring clamps and a rubber O-ring (Figure 8). The exposed surface area for each cell during electrochemical testing was measured to be $706.86 \mathrm{~mm}^{2} .7 .5 \mathrm{ml}$ of corrosive electrolyte was poured into each cell. The mercury/mercurous sulphate electrodes were held in place using rubber corks (see Figure 8, ventilation holes were drilled in the corks). The SCEs were held in place using laboratory stands.

Immediately after the electrolyte was poured into the cells, the experimental setup was connected to a Gammry Reference 600 potentiostat in the SS configuration (the LREs were connected to the working electrode terminals and the corroding Q-panel sample was connected to the reference electrode terminal, as per Figures 2 and 4). ENM data was acquired for 34 minutes and 8 seconds at a sampling frequency of $2 \mathrm{~Hz}$, yielding 4096 data points total. This data was then separated into eight blocks of 512 data points each. ENANALIZ, developed by Cottis, was then used to linearly detrend this data and obtain the standard deviation and mean of the current and potential noise signals for each data block. The noise resistance of each data block was then calculated, as per eq.17. Though linear detrending of ENM data to remove DC drift has been criticised [8], it was considered adequate for the present experiment. This reasoning is based on the simple nature of the corroding system consistently used in each experiment, and prior use of the ENANALIZ 
software in the literature for similar experiments [17,35]. Ultimately, the corroding Q-panel is present simply to provide a source of significant current noise. Measuring the attenuation of this noise by the LREs is the goal of the experiment.

A control experiment utilising the standard SB technique was also performed; the cell setup was similar to that for the SS technique except that two separate sections of Q-panel were connected to the working electrode terminals, and a hollow Perspex tube connected the two cells forming the salt bridge. In this instance, $15 \mathrm{ml}$ of corrosive electrolyte was used and a single mercury/mercurous sulphate electrode provided a stable reference potential.

The AC impedance of each LRE was measured via electrochemical impedance spectroscopy (EIS) using a Gammry Reference 600 potentiostat. The LREs were connected to the working electrode terminal and a large surface area platinum wire mesh was connected to the reference and counter electrode terminals. Both electrodes were immersed in $0.1 \mathrm{M}$ $\mathrm{NaCl}$ solution. An oscillating potential of $+/-10 \mathrm{mV}$ vs. open circuit potential (OCP) was applied to the working electrode terminal, from a frequency of $1,000,000 \mathrm{~Hz}$ through to $0.001 \mathrm{~Hz}$, with 10 data points per decade, and the AC response was measured.

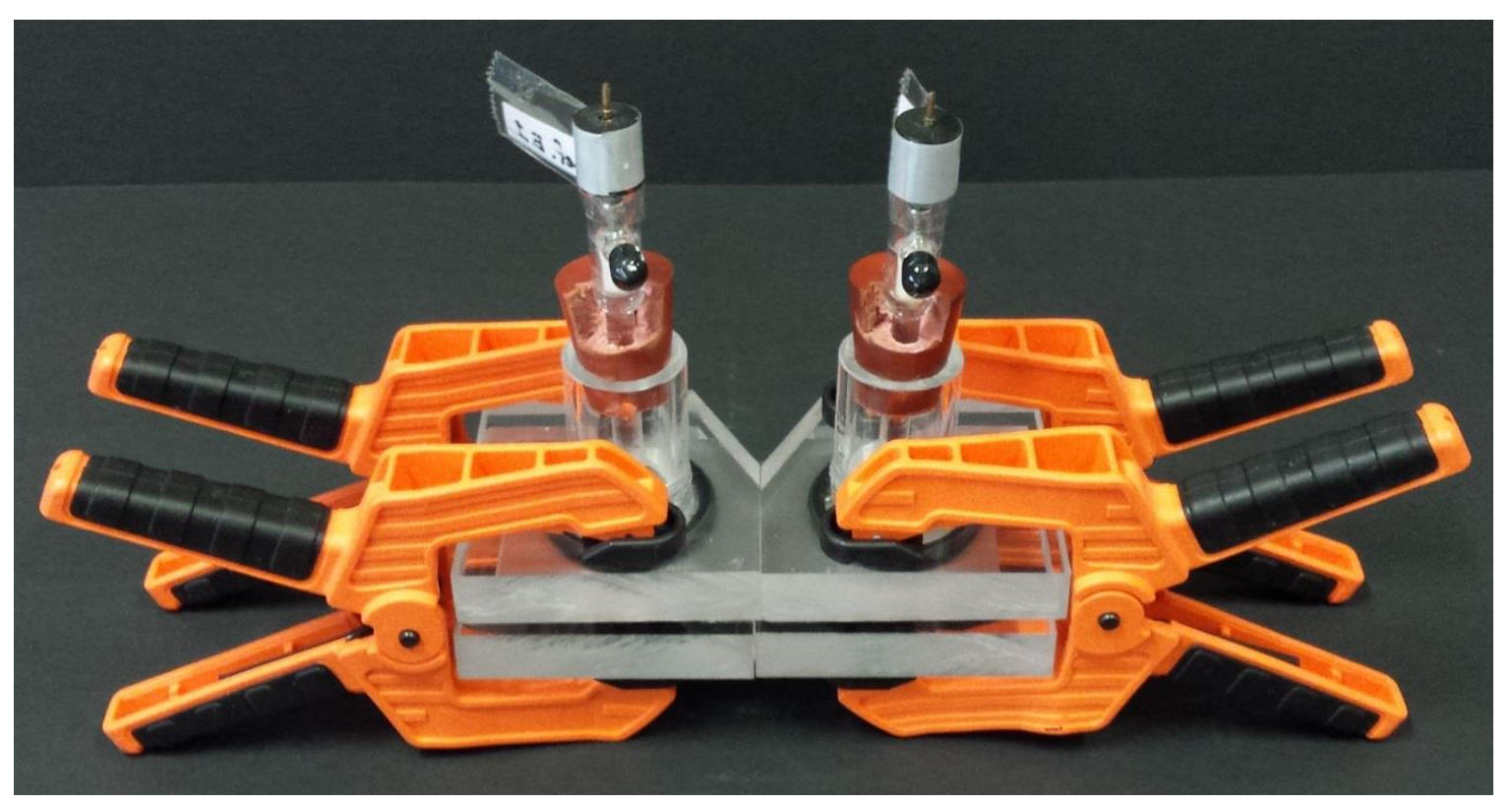

Figure 8: Photograph showing experimental setup for the SS technique.

\subsection{Results and Discussion}

As outlined previously, reference electrode impedance has no effect on ENM data for the SB technique. Thus, the noise resistance obtained by this technique and presented in 
Figure 9 can be considered the minimum noise resistance obtainable for the corroding system analysed. The noise resistance data is what would be expected of a bare corroding metal; a relatively low noise resistance initially followed by a rapid increase (of approximately one order of magnitude) to a steady state, likely due to the formation of corrosion products on the surface [38].

It can be seen in Figure 9 that the choice of LRE had a significant impact on the calculated noise resistance when using the SS technique. The use of SCEs appears to have had a measurable but minimal impact on the noise resistance, implying that these electrodes are relatively low impedance. By contrast, the mercury/mercurous sulphate electrodes had a significant impact on the noise resistance, with an increase of more than two orders of magnitude measured in some instances. Also of great interest, the combination of Ref.E5 and Ref.E6 produced a noise resistance approximately one order of magnitude higher than the combination of Ref.E3 and Ref.E4, despite all four electrodes being of the same type and design, and from the same manufacturer. For all of the LREs, a similar trend in the noise resistance over time was observed relative to the results obtained using the SB technique.

Of critical importance, the trends observed in the noise resistance plots (Figure 9) due to changes in LRE are repeated in the standard deviation of current noise plots (Figure 10), but in reverse. Conversely, there is minimal change in the standard deviation of potential noise plots (Figure 11). This demonstrates experimentally that calculated standard deviation of current noise is inversely proportional to the impedance of the recording reference electrodes, whilst calculated noise resistance is directly proportional to the impedance of said electrodes, as dictated by eq. 8 and eq. 22 respectively. The impedance of the recording reference electrodes attenuates measured current noise in the SS technique, and thus adds to the calculated noise resistance. Potential noise is unaffected by the impedance of said electrodes as per eq.13.

Although there was limited change in the standard deviation of potential noise between the different LREs used, it is clear that there is still a measurable and reproducible difference between them (Figure 11). This would at first appear to be in contradiction to eq.13. However, it can be seen from the standard deviation of potential noise plots that any measured difference is between the different types of LREs used, i.e. mercury/mercurous sulphate and SCE. As stated earlier, the combination of Ref.E5 and Ref.E6 produced a noise resistance approximately one order of magnitude higher than the combination of Ref.E3 and 
Ref.E4, but both pairs of electrodes produced an identical standard deviation of potential noise. ENM performed using the SB technique with Ref.E5 also produced an identical standard deviation of potential noise. Thus it is likely that the measured difference in potential noise power is dependent on the type of LRE used and is independent of electrode impedance.

It can be seen in the mean potential noise plots (Figure 12) that use of the SS technique has reversed the polarity of the potential measurements, consistent with the theoretical model developed in Section 2.

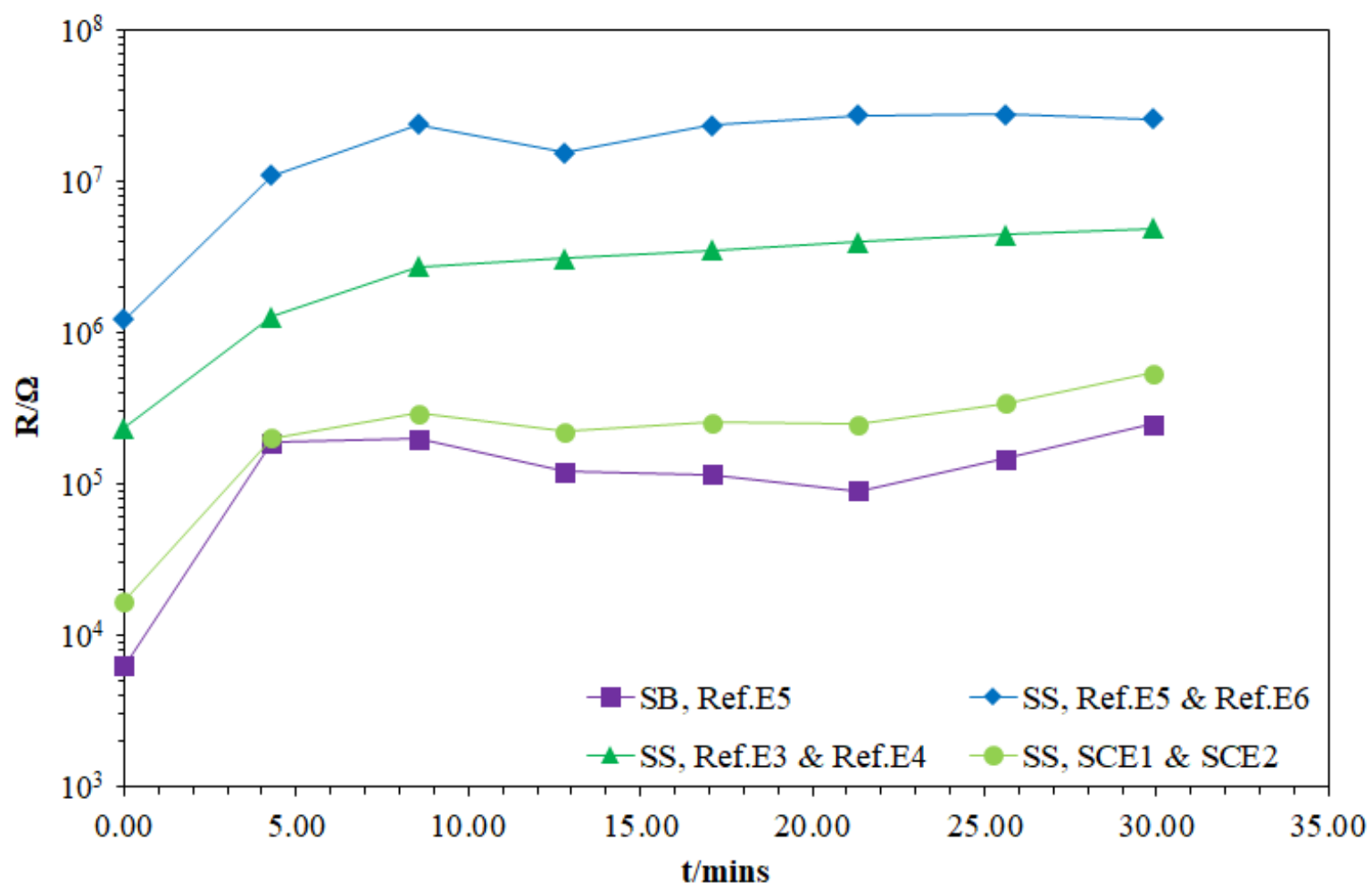

Figure 9: Noise resistance vs. time plot for ENM performed with (a) salt bridge technique using Ref.E5 (๘), (b) single substrate technique using Ref.E3 and Ref.E4 ( $\mathbf{\Delta})$, (c) single substrate technique using Ref.E5 and Ref.E6 (•), and (d) single substrate technique using SCE1 and SCE2 (•). 


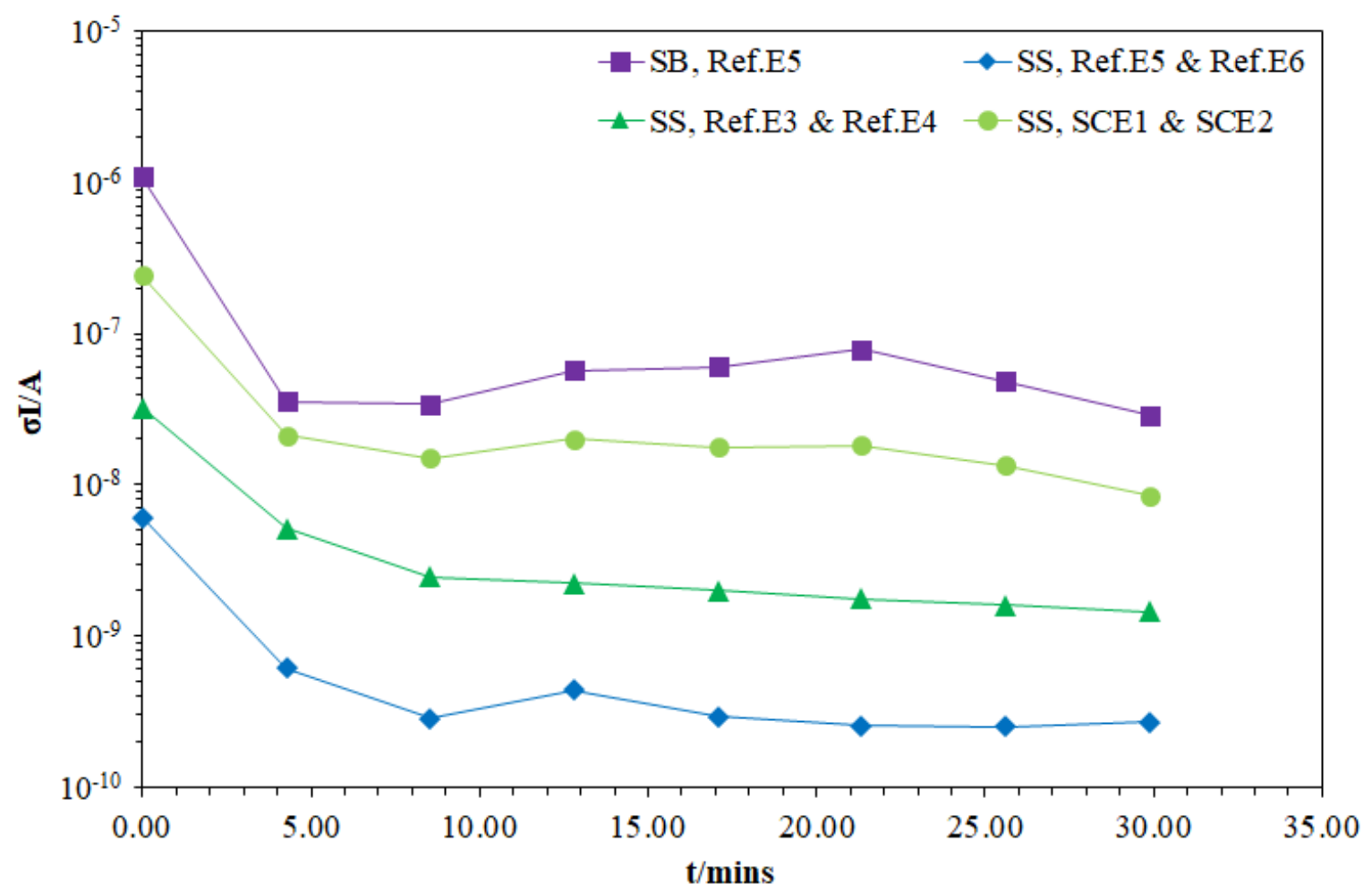

Figure 10: Standard deviation of current noise vs. time plot for ENM performed with (a) salt bridge technique using Ref.E5 (๘), (b) single substrate technique using Ref.E3 and Ref.E4 ( $\mathbf{\Delta})$, (c) single substrate technique using Ref.E5 and Ref.E6 ( $\bullet$ ), and (d) single substrate technique using SCE1 and SCE2 (•).

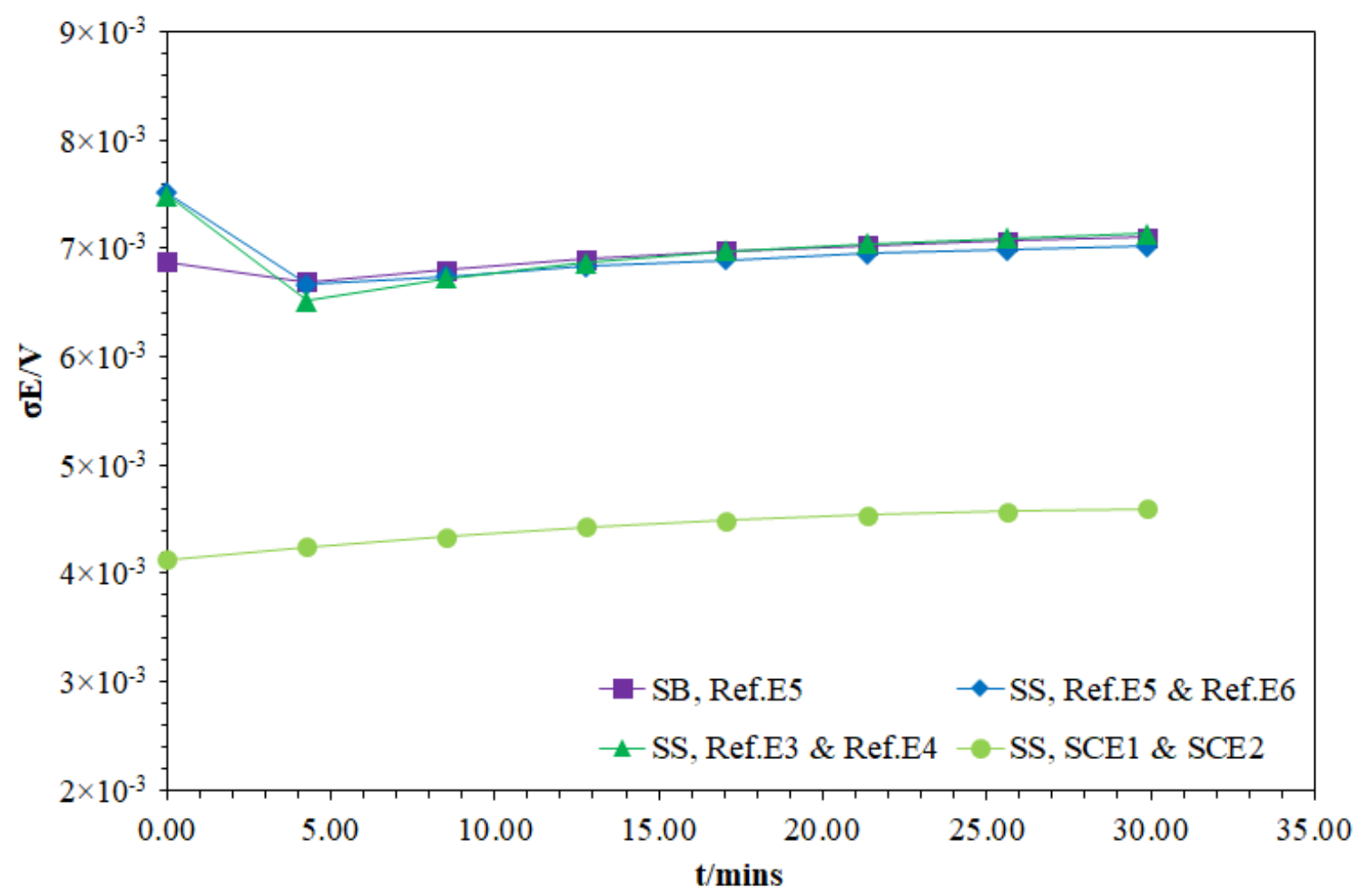

Figure 11: Standard deviation of potential noise vs. time plot for ENM performed with (a) salt bridge technique using Ref.E5 (-), (b) single substrate technique using Ref.E3 and Ref.E4 ( $\mathbf{\Delta}$ ), (c) single 
substrate technique using Ref.E5 and Ref.E6 ( $\bullet$ ), and (d) single substrate technique using SCE1 and SCE2 $(\bullet)$.

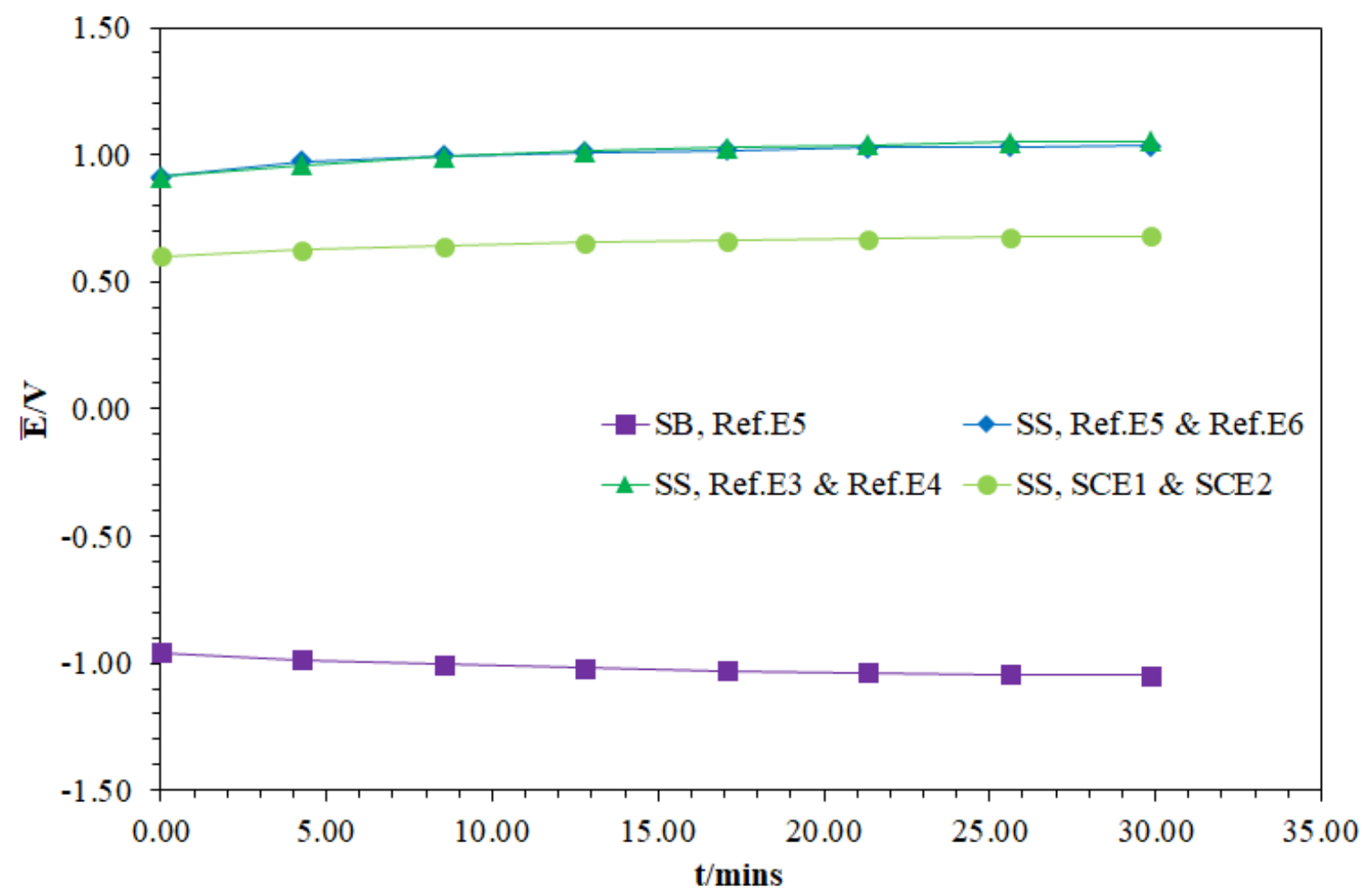

Figure 12: Mean potential noise plot for ENM performed with (a) salt bridge technique using Ref.E5 (一), (b) single substrate technique using Ref.E3 and Ref.E4 ( $\mathbf{\Delta}$ ), (c) single substrate technique using

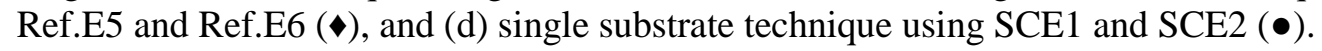

A measurement of the impedance modulus and phase shift of each of the LREs is given in Figures 13 and 14 respectively. Firstly, it is clear that all of the electrodes tested exhibit high impedance in the frequency range $1-100 \mathrm{mHz}$, and especially so in the frequency range $1-10 \mathrm{mHz}$. It is also noteworthy that for all of the electrodes tested, the entire impedance spectrum is above $1 \mathrm{k} \Omega$, the impedance suggested for a "good" LRE in Section 3. Secondly, the phase angle results demonstrate that all of the electrodes exhibited significant capacitive behaviour, with multiple time constants implied by the many inflections in the phase angle plots. Considering this data and the results in Figures 9 and 10, it is likely that both the resistive and capacitive characteristics of the electrodes are responsible for the consistently higher noise resistance measured via the SS technique relative to the SB technique. Of critical importance however, capacitive effects are beyond the scope of the strictly linear EEC that the theoretical model presented herein is based on. Therefore, the 
exact magnitude of the impedance contribution of each individual electrode to the measured noise resistance cannot be determined.

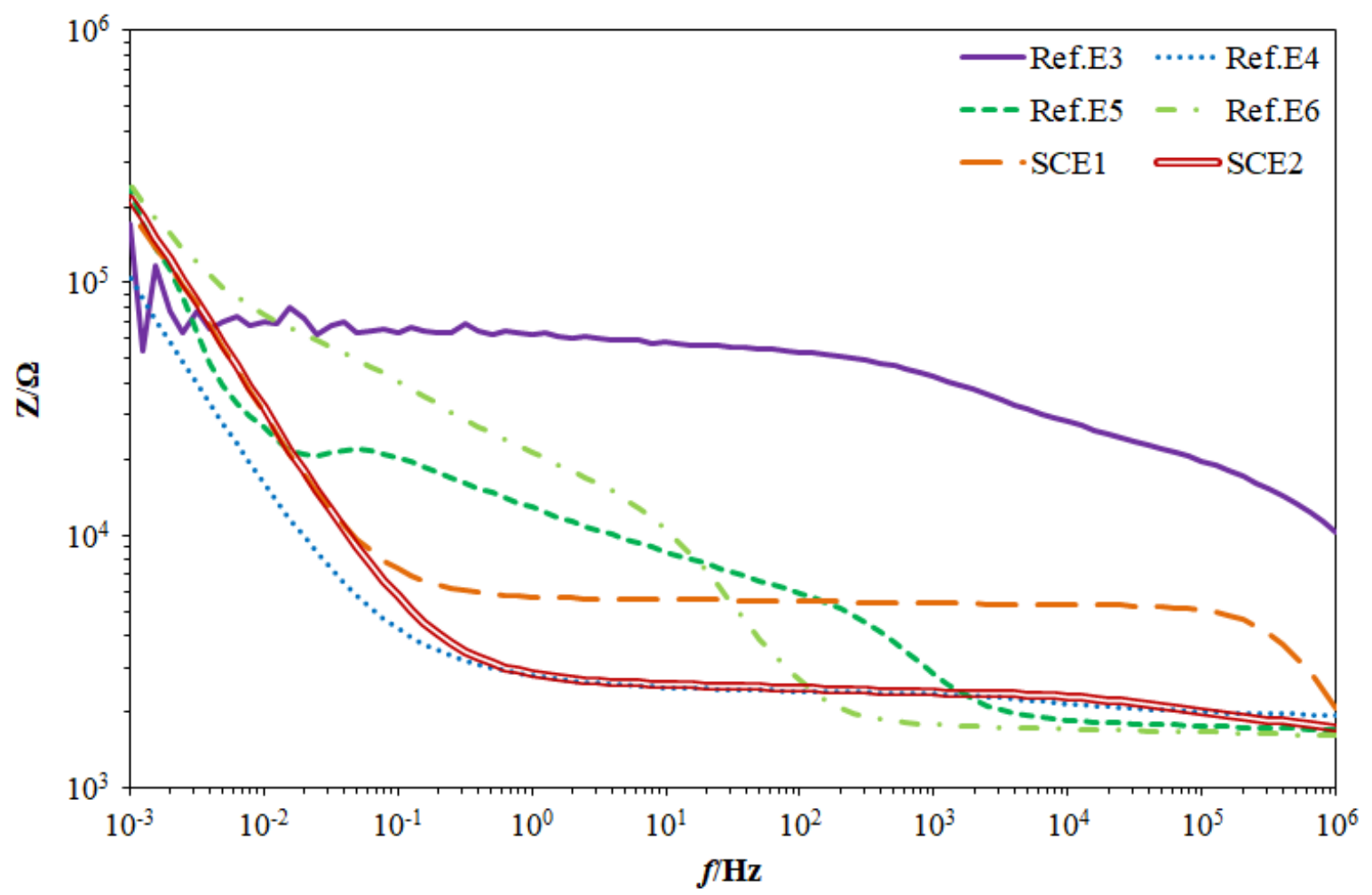

Figure 13: EIS impedance plots for laboratory reference electrodes (a) Ref.E3, (b) Ref.E4, (c) Ref.E5, (d) Ref.E6, (e) SCE1, and (f) SCE2, used in electrochemical noise measurement experiments. 


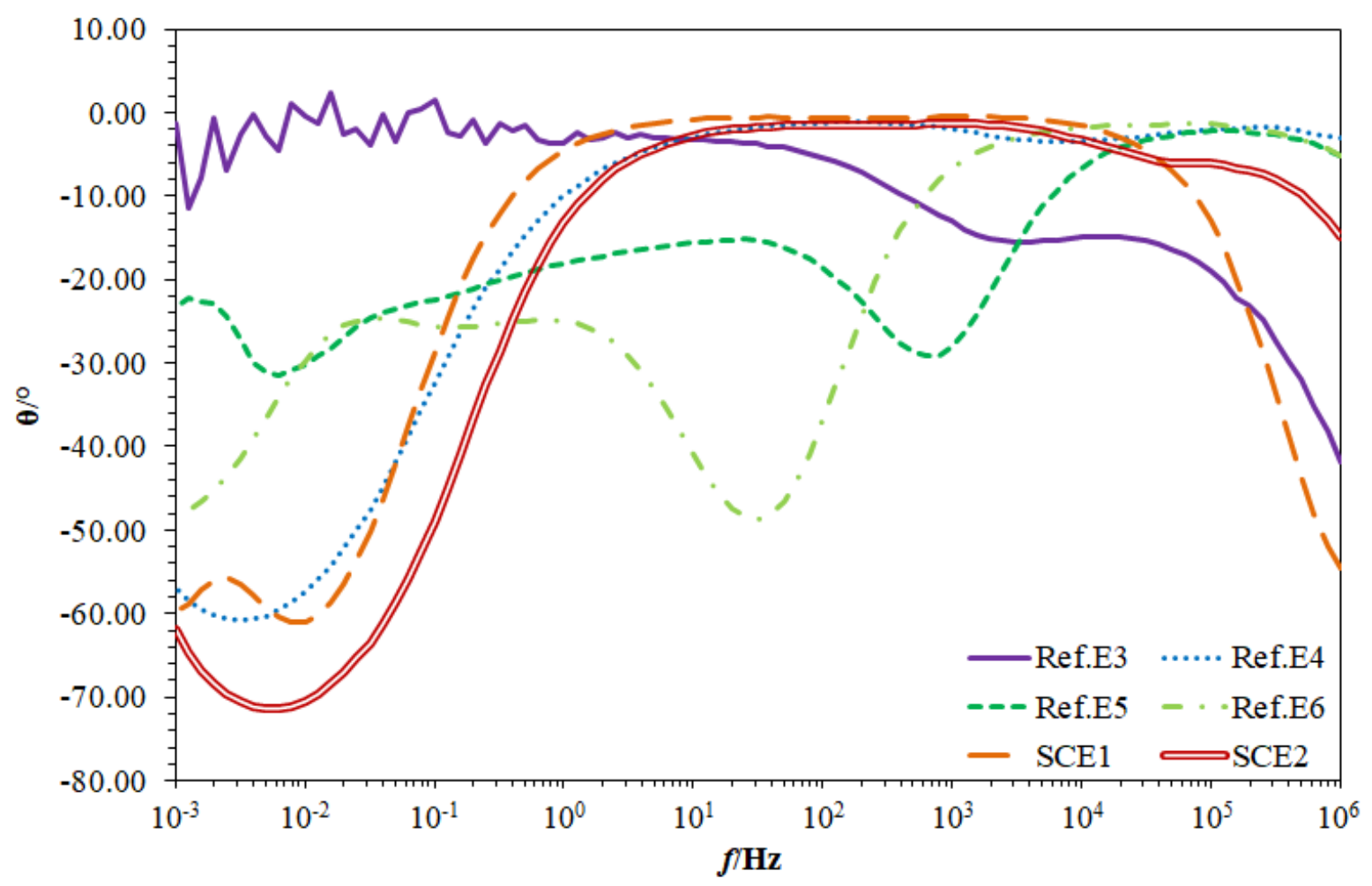

Figure 14: EIS phase angle plots for laboratory reference electrodes (a) Ref.E3, (b) Ref.E4, (c) Ref.E5, (d) Ref.E6, (e) SCE1, and (f) SCE2, used in electrochemical noise measurement experiments.

\section{Conclusion}

A theoretical model for the single substrate (SS) technique of electrochemical noise measurement (ENM) was developed, and the effect of connecting laboratory reference electrodes (LREs) to the working electrode terminals of the recording instrument was determined. According to the theoretical model, when using two identical LREs of sufficiently low impedance in solutions with negligible resistance, the noise resistance obtained using the SS configuration is equivalent to that obtained using the salt bridge (SB) configuration, theoretically validating the SS configuration. The theoretical model also demonstrated that should high impedance LREs be utilised with the SS technique, the impedance of the two recording LREs will attenuate the electrochemical current noise and hence add to the noise resistance.

In addition, the experimental work presented herein has demonstrated that LREs can have significantly high impedance and capacitance, and that these properties can impact ENM data obtained via the SS technique. The experimental analysis was consistent with the 
theoretical model, demonstrating the need to measure the impedance of LREs in the laboratory prior to performing ENM in the field.

\section{Acknowledgements}

The authors gratefully acknowledge Joe Williams, Abhishek Saxena and Jason Hodges for their support and helpful technical advice, the Australian Research Council Industrial Transformation Research Hubs Scheme (Project Number IH130100017) for funding this research, as well as the financial and technical support provided by BlueScope Limited and the University of Wollongong, Australia.

\section{References}

[1] B.S. Skerry, D.A. Eden, T. Sherwin-williams, P. Centre, Electrochemical testing to assess corrosion protective coatings, Prog. Org. Coatings. 15 (1987) 269-285.

[2] B.S. Skerry, D.A. Eden, Characterisation of coatings performance using electrochemical noise analysis, Prog. Org. Coatings. 19 (1991) 379-396.

[3] S.S. Jamali, D.J. Mills, A critical review of electrochemical noise measurement as a tool for evaluation of organic coatings, Prog. Org. Coatings. 95 (2016) 26-37. doi:10.1016/j.porgcoat.2016.02.016.

[4] J.R. Kearns, J.R. Scully, P.R. Roberge, D.L. Reichert, J.L. Dawson, Electrochemical Noise Measurement for Corrosion Applications, American Society for Testing and Materials, West Conshohocken, 1996.

[5] S. Mabbutt, D.J. Mills, C.P. Woodcock, Developments of the electrochemical noise method (ENM) for more practical assessment of anti-corrosion coatings, Prog. Org. Coatings. 59 (2007) 192-196. doi:10.1016/j.porgcoat.2006.09.017.

[6] J.F. Chen, W.F. Bogaerts, Electrochemical Emission Spectroscopy for Monitoring Uniform and Localized Corrosion, Corrosion. 52 (1996) 753-759. doi:10.5006/1.3292068.

[7] D.-H. Xia, S.-Z. Song, Y. Behnamian, Detection of corrosion degradation using electrochemical noise $(\mathrm{EN})$ : review of signal processing methods for identifying corrosion forms, Corros. Eng. Sci. Technol. 2782 (2016) 1-18. doi:10.1179/1743278215Y.0000000057.

[8] D.-H. Xia, Y. Behnamian, Electrochemical noise: a review of experimental setup, instrumentation and DC removal, Russ. J. Electrochem. 51 (2015) 593-601. doi:10.1134/S1023193515070071. 
[9] C. Ma, S. Song, Z. Gao, J. Wang, W. Hu, Y. Behnamian, D.H. Xia, Electrochemical noise monitoring of the atmospheric corrosion of steels: identifying corrosion form using wavelet analysis, Corros. Eng. Sci. Technol. 52 (2017) 432-440. doi:10.1080/1478422X.2017.1320117.

[10] S.-Z. Song, W.-X. Zhao, J.-H. Wang, J. Li, Z.-M. Gao, D.-H. Xia, Field Corrosion Detection of Nuclear Materials using Electrochemical Noise Techinique, Prot. Met. Phys. Chem. Surfaces. 54 (2018) 340-346. doi:10.1134/S2070205118020211.

[11] R. Zhao, D.H. Xia, S.Z. Song, W. Hu, Detection of SCC on 304 stainless steel in neutral thiosulfate solutions using electrochemical noise based on chaos theory, AntiCorrosion Methods Mater. 64 (2017) 241-251. doi:10.1108/ACMM-10-2015-1581.

[12] S.J. Mabbutt, D.J. Mills, Novel configurations for electrochemical noise measurements, Br. Corros. J. 33 (1998) 158-160.

[13] D.J. Mills, S.J. Mabbutt, Developments in the electrochemical noise method to assess anti-corrosive coatings and inhibitors, in: 7th Int. Symp. Electrochem. Methods Corros. Res., 2000.

[14] S. Mabbutt, G. Bierwagen, D. Mills, New experimental arrangement for the acquisition of electrochemical noise data from high resistance organic anti-corrosive coatings, Anti-Corrosion Methods Mater. $49 \quad$ (2002) 264-269. doi:10.1108/00035590210431773.

[15] S.J. Mabbutt, D.J. Mills, Recent UK work investigating anti-corrosive organic coatings using the electrochemical noise method (ENM), Surf. Coatings Int. 84 (2001) 277283. http://www.springerlink.com/index/865T9RGW48410J28.pdf.

[16] R.A. Cottis, The significance of electrochemical noise measurements on asymmetric $\begin{array}{lllll}\text { electrodes, } & \text { Electrochim. } & \text { Acta. } & 52 & \text { (2007) }\end{array}$ doi:10.1016/j.electacta.2006.12.042.

[17] S.S. Jamali, D.J. Mills, J.M. Sykes, Measuring electrochemical noise of a single working electrode for assessing corrosion resistance of polymer coated metals, Prog. Org. Coatings. 77 (2014) 733-741. doi:10.1016/j.porgcoat.2013.12.014.

[18] N.G. Thompson, J.H. Payer, Corrosion Testing Made Easy: DC Electrochemical Test Methods, NACE International, Houston, 1998.

[19] D.J.G. Ives, G.J. Janz, Reference Electrodes: Theory and Practice, Second Edi, Academic Press, Inc., New York, 1961.

[20] I. Gamry Instruments, Gamry Technical Note: Reference Electrodes, 2016.

[21] R. Cottis, S. Turgoose, Corrosion Testing Made Easy: Electrochemical Impedance and Noise, NACE International, Houston, 1999.

[22] G. Fafilek, The use of voltage probes in impedance spectroscopy, Solid State Ionics. 176 (2005) 2023-2029. doi:10.1016/j.ssi.2004.06.024.

[23] A. Tran, F. Huet, K. Ngo, P. Rousseau, Artefacts in electrochemical impedance measurement in electrolytic solutions due to the reference electrode, Electrochim. Acta. 56 (2011) 8034-8039. doi:10.1016/j.electacta.2010.12.088.

[24] G. Hsieh, S.J. Ford, T.O. Mason, L.R. Pederson, Experimental limitations in impedance spectroscopy: Part I - simulation of reference electrode artifacts in threepoint measurements, Solid State Ionics. 91 (1996) 191-201.

[25] G. Hsieh, T.O. Mason, L.R. Pederson, Experimental limitations in impedance 
spectroscopy: Part II - electrode artifacts in three-point measurements on Pt/YSZ, Solid State Ionics. 91 (1996) 203-212.

[26] O. Poupard, A.A. Mokhtar, P. Dumargue, Impedance spectroscopy in reinforced concrete: Procedure for monitoring steel corrosion, J. Mater. Sci. 38 (2003) 28452850 .

[27] S. Chechirlian, P. Eichner, M. Keddam, H. Takenouti, H. Mazille, A Specific Aspect of Impedance Measurements in Low Conductivity Media. Artefacts and their Interpretations, Electrochim. Acta. 35 (1990) 1125-1131.

[28] K.N. Allahar, Q. Su, G.P. Bierwagen, D.-H. Lee, Monitoring of the AC-DC-AC Degradation of Organic Coatings Using Embedded Electrodes, Corrosion. 64 (2008) 773-787.

[29] G.P. Bierwagen, X. Wang, D.E. Tallman, In situ study of coatings using embedded electrodes for ENM measurements, Prog. Org. Coatings. 46 (2003) 163-175.

[30] Q. Su, K. Allahar, G. Bierwagen, Embedded electrode electrochemical noise monitoring of the corrosion beneath organic coatings induced by ac-dc-ac conditions, Electrochim. Acta. 53 (2008) 2825-2830. doi:10.1016/j.electacta.2007.10.063.

[31] V. Upadhyay, K.N. Allahar, G.P. Bierwagen, Environmental humidity influence on a topcoat/Mg-rich primer system with embedded electrodes, Sensors Actuators B. Chem. 193 (2014) 522-529. doi:10.1016/j.snb.2013.11.084.

[32] K.N. Allahar, D. Wang, D. Battocchi, G.P. Bierwagen, S. Balbyshev, Real-Time Monitoring of a United States Air Force Topcoat / Mg-Rich ..., Corrosion. 66 (2010) 75003-1-75003-11.

[33] G.P. Bierwagen, K.N. Allahar, Q. Su, V.J. Gelling, Electrochemically characterizing the ac-dc-ac accelerated test method using embedded electrodes, Corros. Sci. 51 (2008) 95-101. doi:10.1016/j.corsci.2008.09.023.

[34] R. a. Cottis, S. Turgoose, J. Mendoza-Flores, The Effects of Solution Resistance on Electrochemical Noise Resistance Measurements: A Theoretical Analysis, (1996) 93100.

[35] S.S. Jamali, D.J. Mills, R. a. Cottis, T.Y. Lan, Analysis of electrochemical noise measurement on an organically coated metal, Prog. Org. Coatings. 96 (2015) 52-57. doi:10.1016/j.porgcoat.2016.01.017.

[36] U. Bertocci, C. Gabrielli, F. Huet, M. Keddam, Noise Resistance Applied to Corrosion Measurements I. Theoretical Analysis, J. Electrochem. Soc. 144 (1997) 2772-2778. doi:10.1149/1.1837896.

[37] U. Bertocci, C. Gabrielli, F. Huet, M. Kecidam, P. Rousseau, Noise Resistance Applied to Corrosion Measurements II. Experimental Tests, J. Electrochem. Soc. 144 (1997) 2779-2785. doi:10.1149/1.1837896.

[38] W.S. Tait, An Introduction to Electrochemical Corrosion Testing for Practicing Engineers and Scientists, 1994. 